\title{
Development of Deflection Angle Stabilizing System for Balancing Robot
}

\author{
Dmitry Fedorov, Andrey Ivoilov, Vadim Zhmud and Vitaly Trubin
}

\author{
Novosibirsk State Technical University, Russia
}

\begin{abstract}
This paper performs determination of a mathematical model of the balancing robot, its linearization and algorithm for calculation of stabilization of the deflection angle from the vertical modal method. This algorithm also makes determination of the angle of deviation from the use of either alpha-beta filter and the angle of rotation of the wheels by means of encoders.
\end{abstract}

Keywords: Balancing robot, automatic control system, the modal synthesis method, the complementary filter, alpha-beta filter, accelerometer, gyroscope, and encoder.

\section{INTRODUCTION}

This paper describes the result of the development of stabilization system for two-wheeled balancing robot. Figure 1 shows the appearance of the robot. This device is mechanical part and platform containing the control electronics of the robot and sensors. Stators of DC motors are rigidly attached to the platform. At the motor rotors, two wheels are mounted. The idea of the device is to maintain the vertical position of the robot by rotating of the wheels, supporting the balance by feedback.

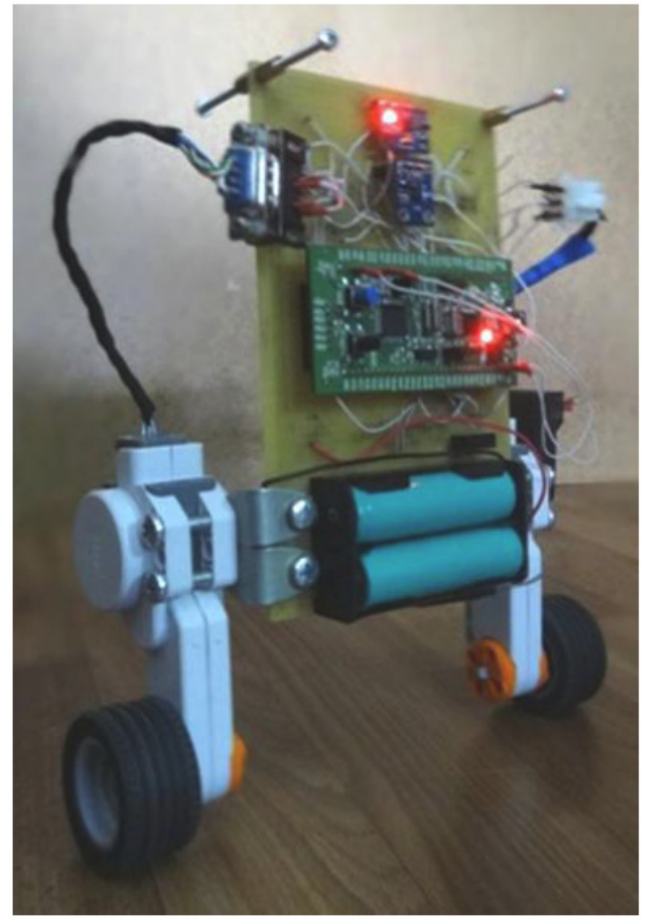

Figure 1: The two-wheeled balancing robot.

*Address correspondence to this author at the Novosibirsk State Technical University and Geophysical Service of SB RAS, Russia; Tel: +7-961-875-1917; Fax: +7-383-3461119; E-mail: oao_nips@bk.ru
Balancing robot is from a mathematical point of view inverted pendulum with a suspension point on the wheel (Figure 2) [1]. It is nonlinear unstable system, which can serve for testing and comparison of different control algorithms. Many unstable systems contain units, representing an inverted pendulum. For example, vehicle Segway, the two-legged walking mechanisms and exoskeletons. Therefore, obtained in the paper results can be widely applyed.

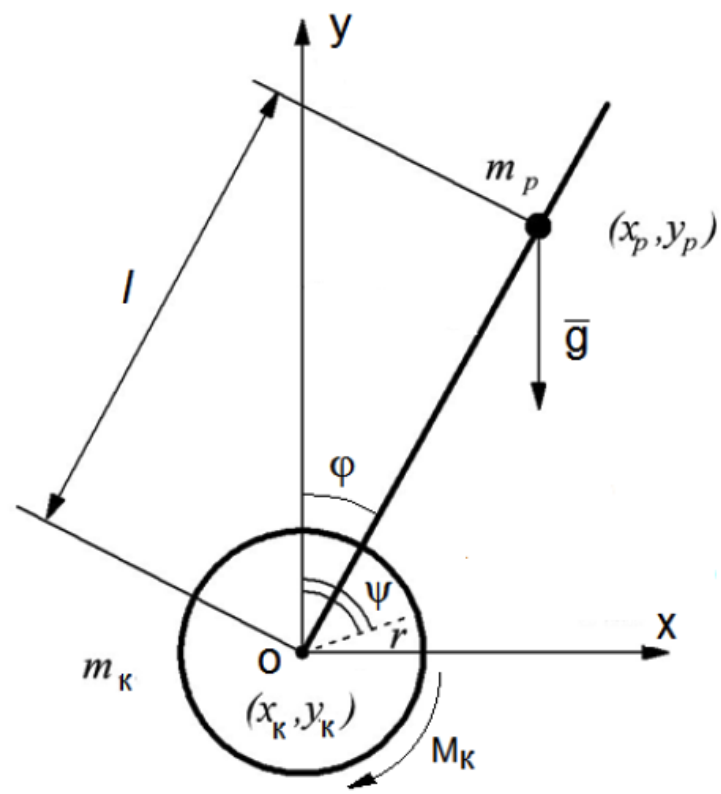

Figure 2: The pendulum with suspension point on the wheel: $O$ is the point of suspension, $M_{\mathrm{k}}$ is moment of the wheel, $\psi$ is angle of rotation of the wheel, $r$ is radius of the wheel, $m_{\mathrm{k}}$ is wheel mass, $\left(x_{k}, y_{k}\right)$ is the center of the wheel mass, $\varphi$ is angle of inclination, $\left(x_{p}, y_{p}\right)$ is the center of mass of the robot, $I$ is the distance from the center of mass of the robot to the suspension point (center of mass of the wheel) $\bar{g}$ is free fall acceleration.

The primary problem is that the definition of the mathematical model of the object, as well as checking the solvability of the task of the control design. 
An ssensial part of any system of automatic control is mesn for the measurement of the output value. The accuracy of this measurement depends on the quality of regulation and the stability margin. This paper discusses the features of the accelerometer and gyroscope included in the control system MPU6050, and suggested to combine the testimony of these two sensors using complementary filter that allows you to accurately determine the deflection angle at the current time.

The result of the researche is stabilization system for balancing robot, which has been calculated by means of modal method. This system controls the state of the robot in a vertical position and can work off external disturbances.

\section{THE RESULTING MATHEMATICAL MODEL OF OBJECT}

Before the start of the development of the stabilization system, it is necessary to find out what is the object of control in terms of the mathematical model of signal transformation. To build a system of stabilization it is necessary to clarify what values it is required to measure or evaluate and in what mode the system works. Based on this information, you want to select a control method, the method of calculation of the controller (regulator) and method of estimating unknown quantities.

To obtain a mathematical model of the object you must compose the system of differential equations describing the physical processes in it. To solve this problem we use the Lagrange equation of the second kind:

$\frac{d}{d t}\left(\frac{d T}{d \dot{q}_{i}}\right)-\frac{d T}{d q_{i}}=Q_{i}$.

Here $T$ is the kinetic energy of the system, $Q_{\mathrm{i}}$ is generalized force, $q_{\mathrm{i}}$ are the generalized coordinates.

In this case, the generalized coordinates are the angle of inclination $\varphi$ and wheel rotation angle $\psi$. The kinetic energy of the system is as follows:

$T=2 T_{k}+T_{p}$.

Here $T_{\mathrm{k}}$ is the kinetic energy of the wheels; $T_{p}$ is the kinetic energy of the robot.

The total kinetic energy of each of the components includes a rotational and onward energy:

$$
\begin{aligned}
& T_{\kappa}=T_{\kappa . \beta p .}+T_{\kappa . n o c .}=\frac{J_{\kappa} \psi^{2}}{2}+\frac{m_{\kappa} v_{\kappa}^{2}}{2}= \\
& =\frac{\rho^{2} m_{\kappa} \psi^{2}}{2}+\frac{m_{\kappa} r^{2} \psi^{2}}{2}=\frac{m_{\kappa} \psi^{2}\left(r^{2}+\rho^{2}\right)}{2}, \\
& T_{p}=T_{p . \beta p .}+T_{p . n o c .}=\frac{J_{p} \psi^{2}}{2}+\frac{m_{p} v_{p}^{2}}{2} .
\end{aligned}
$$

Here $J_{K}$ and $J_{p}$ are moments of inertia of the wheel and the robot, respectively, $\rho$ is radius of inertia of the wheel.

The forward speed of the robot is determined by the speed of its center of mass:

$v_{p}^{2}=v_{p_{-} x}^{2}+v_{p_{-} y}^{2}=\dot{x}_{p}^{2}+\dot{y}_{p}^{2}$,

Since $y_{k}=0$, coordinates of the center of mass of the pendulum rod and wheel center are related by:

$$
\begin{aligned}
& x_{p}=x_{k}+l \sin \varphi, \text { consequently } \\
& \dot{x}_{p}=\dot{x}_{k}+l \cdot \dot{\varphi} \cos \varphi=r \dot{\psi}+l \cdot \dot{\varphi} \cos \varphi, \\
& \dot{x}_{p}^{2}=r^{2} \dot{\psi}^{2}+2 r \dot{\psi} \cdot l \cdot \dot{\varphi} \cos \varphi+l^{2} \cdot \dot{\varphi}^{2} \cos ^{2} \varphi \\
& y_{p}=l \cos \varphi, \text { consequently } \\
& \dot{y}_{p}=-l \cdot \dot{\varphi} \sin \varphi, \\
& \dot{y}_{p}^{2}=l^{2} \cdot \dot{\varphi}^{2} \sin ^{2} \varphi .
\end{aligned}
$$

Then the velocity of the center of mass of the robot:

$$
\begin{aligned}
& v_{p}^{2}=r^{2} \psi^{2}+2 r l \dot{\psi} \dot{\varphi} \cos \varphi+l^{2} \cos ^{2} \varphi \cdot \dot{\varphi}^{2}+ \\
& +l^{2} \sin ^{2} \varphi \cdot \dot{\varphi}^{2}= \\
& =r^{2} \psi^{2}+2 r l \dot{\psi} \dot{\varphi} \cos \varphi+l^{2} \dot{\varphi}^{2} .
\end{aligned}
$$

As a result, the kinetic energy of the robot will be the following:

$T_{v}=\frac{\lambda^{2} m_{v} \varphi^{2}}{2}+$

$+\frac{m_{v}}{2}\left(r^{2} \psi^{2}+2 r l \dot{\psi} \dot{\varphi} \cos \varphi+l^{2} \dot{\varphi}^{2}\right)$.

Here $\lambda$ is the radius of gyration of robot relatively the axis of the wheels. In view of (2) and (3) the kinetic energy of the system will be equal to: 
$T=m \kappa \psi^{2}\left(\rho^{2}+r^{2}\right)+$

$+\frac{m_{p}}{2}\left(\lambda^{2} \varphi^{2}+2 r l \dot{\psi} \dot{\varphi} \cos \varphi+l^{2} \dot{\varphi}^{2}\right)$.

We got the first differential equation system in accordance with (1). After the differentiating of (4), along $\varphi, \dot{\varphi}$ and $t$ we get:

$\frac{d T}{d \dot{\varphi}}=\frac{m_{v}}{2}\left(2 \lambda^{2} \dot{\varphi}+2 r l \dot{\psi} \cos \varphi+l^{2} \dot{\varphi}\right)$,

$\frac{d}{d t}\left(\frac{d T}{d \dot{\varphi}}\right)=\frac{m_{v}}{2}\left(2 \lambda^{2} \ddot{\varphi}+2 r l(\ddot{\psi} \cos \varphi-\dot{\psi} \sin \varphi \cdot \dot{\varphi})+l^{2} \ddot{\varphi}\right)$,

$\frac{d T}{d \varphi}=-\frac{m_{v}}{2} \cdot 2 r l \dot{\psi} \sin \varphi \cdot \dot{\varphi}=-m_{v} r l \dot{\psi} \sin \varphi \cdot \dot{\varphi}$

$\frac{d}{d t}\left(\frac{d T}{d \dot{\varphi}}\right)-\frac{d T}{d \varphi}=m_{v}\left(\lambda^{2}+l^{2}\right) \ddot{\varphi}+m_{v} r l \ddot{\psi} \cos \varphi=$

$=m_{v} g l \sin \varphi-2 M_{K}$.

Here $M_{K}$ is moment acting on the platform of the motor side. We get second times differential equation of the system in accordance with (1), differentiating (4), along $\varphi, \dot{\varphi}$ and $t$ :

$\frac{d T}{d \psi}=2 m_{K} \dot{\psi}\left(\rho^{2}+r^{2}\right)+\frac{m_{v}}{2}\left(2 r^{2} \dot{\psi}+2 r l \dot{\varphi} \cdot \sin \varphi\right)$,

$\frac{d}{d t}\left(\frac{d T}{d \dot{\psi}}\right)=2 m_{K} \ddot{\psi}\left(\rho^{2}+r^{2}\right)+m_{v} r^{2} \ddot{\psi}+m_{v} r l\left(\ddot{\varphi} \cos \varphi-\dot{\varphi}^{2} \sin \varphi\right)$,

$\frac{d T}{d \psi}=0$

$\frac{d}{d t}\left(\frac{d T}{d \dot{\psi}}\right)-\frac{d T}{d \psi}=\ddot{\psi}\left(2 m_{K}\left(\rho^{2}+r^{2}\right)+m_{p} r^{2}\right)+$

$+m_{P} r l\left(\ddot{\varphi} \cos \varphi-\dot{\varphi}^{2} \sin \varphi\right)=M_{K}$

The ratios for the DC motor are of the form: $я$

$\omega=\frac{U-I_{Я} R_{Я}}{k_{e}}$

$M_{\text {дв }}=K_{m} I_{\text {Я }} ;$

$J_{\text {дВ }} \varepsilon=M_{\text {дВ }}-M_{\text {НАГ }}$.

Here $M_{д B}$ is moment generated by motor $M_{\mathrm{HA \Gamma}}$ is load moment, $J_{\mathrm{AB}}$ is the moment of inertia of the motor, $\omega$ is angular velocity of rotation of the rotor relatively to the stator, $\varepsilon$ is the angular acceleration of the rotor, $U$,
$I_{\text {Я }}, R_{\text {Я }}$ are voltage, current and resistance of the rotor chain, $k_{\mathrm{m}}, k_{\mathrm{e}}$ are structural coefficients $k_{\mathrm{m}}$ includes transfer coefficient of wheel gear coupling with the motor shaft.

From the equation (8) we express the current in the rotor circuit and substitute it into (7):

$\omega=\frac{U}{k_{e}}-\frac{M_{\text {дВ }} R_{\text {Я }}}{k_{m} k_{e}}$.

The expression for the moment of the motor will be as follows:

$M_{\text {дв }}=\frac{k_{m} U}{R_{\text {Я }}}-\frac{k_{m} k_{e}}{R_{\text {Я }}} \omega$.

Substituting (11) into (9) and solve the equation for the load moment:

$$
M_{\text {НАГ }}=\frac{k_{m} U}{R_{\text {Я }}}-\frac{k_{m} k_{e}}{R_{\text {Я }}} \omega-J_{\text {дВ }} \varepsilon .
$$

As far as the platform and wheels are motor load, let $M_{\mathrm{K}}=M_{\mathrm{HA} \Gamma}$. Substituting (12) into (5) and (6), taking into account that $\omega=\dot{\psi}-\dot{\varphi}$ и $\varepsilon=\ddot{\varphi}-\ddot{\psi}$, we have:

$$
\begin{aligned}
& \left(m_{p}\left(\lambda^{2}+l^{2}\right)+2 J_{\text {ДВ }}\right) \ddot{\varphi}+\left(m_{p} r l-2 J_{\text {口В }}\right) \ddot{\psi} \cos \varphi= \\
& =m_{p} g l \sin \varphi+2 \frac{k_{m} k_{e}}{R_{\text {Я }}}(\dot{\psi}-\dot{\varphi})-2 \frac{k_{m} U}{R_{\text {Я }}}, \\
& \ddot{\psi}\left(2\left(m_{K}\left(\rho^{2}+r^{2}\right)+\frac{1}{2} m_{p} r^{2}\right)+J_{\text {口В }}\right)+ \\
& +\left(m_{p} r l-J_{\text {ДВ }}\right)(\ddot{\varphi} \cos \varphi-\dot{\varphi} \sin \varphi)=\frac{k_{m} U}{R_{\text {Я }}}-\frac{k_{m} k_{e}}{R_{\text {Я }}}(\dot{\psi}-\dot{\varphi}) .
\end{aligned}
$$

We carry out linearization at the point $\varphi=0$, take into account $\sin \varphi=\varphi, \cos \varphi=1$ and assume $\dot{\varphi}=0$. Neglecting the mass of the wheel, we get:

$$
\begin{aligned}
& \left(m_{p}\left(\lambda^{2}+l^{2}\right)+2 J_{\text {ДВ }}\right) \ddot{\varphi}+\left(m_{p} r l-2 J_{\text {ДB }}\right) \ddot{\psi}= \\
& =m_{p} g l \sin \varphi+2 \frac{k_{m} k_{e}}{R_{\text {Я }}}(\dot{\psi}-\dot{\varphi})-2 \frac{k_{m} U}{R_{\text {Я }}}, \\
& \ddot{\psi}\left(m_{p} r^{2}+J_{\text {मВ }}\right)\left(m_{p} r l-J_{\text {ДB }}\right) \ddot{\varphi}= \\
& =\frac{k_{m} U}{R_{\text {Я }}}-\frac{k_{m} k_{e}}{R_{\text {Я }}}(\dot{\psi}-\dot{\varphi}) .
\end{aligned}
$$

Let

$$
\begin{aligned}
& h_{11}=m_{p}\left(\lambda^{2}+l_{2}\right)+2 J_{\text {ДВ }} ; \\
& h_{12}=m_{p} r l-2 J_{\text {ДВ }} ;
\end{aligned}
$$


$h_{13}=m_{p} r l-J_{\text {дВ }}$;

$h_{14}=m_{p} r^{2}+J_{\text {ДВ }}$;

$h_{21}=2 \frac{k_{m} k_{e}}{R_{\text {g }}} ; h_{22}=m_{p} g l ;$

$h_{23}=\frac{k_{m} k_{e}}{R_{\text {Я }}} ; h_{24}=2 \frac{k_{m} U}{R_{\text {Я }}} ;$

$h_{32}=\frac{k_{m} U}{R_{\text {g }}}$.

Let add the two equations: $\dot{\psi}=\dot{\psi} ; \dot{\varphi}=\dot{\varphi}$.

We introduce state variables:

$x_{1}=\dot{\varphi} ; x_{2}=\varphi ; x=\dot{\psi} ; x_{4}=\psi$.

Then, in the form of a matrix system (13) takes the following form:

$H_{1} \dot{x}=H_{2} x+H_{3} u$

where

$H_{1}=\left[\begin{array}{cccc}h_{11} & 0 & h_{12} & 0 \\ 0 & 1 & 0 & 0 \\ h_{13} & 0 & h_{14} & 0 \\ 0 & 0 & 0 & 1\end{array}\right]$,

$H_{2}=\left[\begin{array}{cccc}-h_{21} & h_{22} & h_{21} & 0 \\ 1 & 0 & 0 & 0 \\ h_{23} & 0 & -h_{23} & 0 \\ 0 & 0 & 1 & 1\end{array}\right]$,

$H_{3}=\left[\begin{array}{c}-h_{31} \\ 0 \\ h_{32} \\ 0\end{array}\right], x=\left[\begin{array}{c}\dot{\varphi} \\ \varphi \\ \dot{\psi} \\ \psi\end{array}\right]$.

To go to the standard description of the system, we multiply (14) on the left at $H_{1}^{-1}$. As a result, we get:

$\dot{x}=A x+B u$

Here

$A=\left[\begin{array}{cccc}-a_{1} & a_{2} & a_{1} & 0 \\ 1 & 0 & 0 & 0 \\ a_{3} & -a_{4} & -a_{3} & 0 \\ 0 & 0 & 1 & 0\end{array}\right], B=\left[\begin{array}{c}-b_{1} \\ 0 \\ b_{2} \\ 0\end{array}\right]$, $a_{1}=\frac{h_{12} h_{23}+h_{14} h_{21}}{h_{11} h_{14}-h_{12} h_{13}}, a_{2}=\frac{h_{14} h_{22}}{h_{11} h_{14}-h_{12} h_{13}}$,

$a_{3}=\frac{h_{11} h_{23}+h_{13} h_{21}}{h_{11} h_{14}-h_{12} h_{13}}, a_{4}=\frac{h_{13} h_{22}}{h_{11} h_{14}-h_{12} h_{13}}$,

$b_{1}=\frac{h_{12} h_{32}+h_{14} h_{31}}{h_{11} h_{14}-h_{12} h_{13}}, b_{2}=\frac{h_{11} h_{32}+h_{13} h_{31}}{h_{11} h_{14}-h_{12} h_{13}}$.

To determine the stability of the system we define its characteristic equation:

$A(p)=\operatorname{det}(p l-A)=$

$=p^{4}+A_{3} p^{3}+A_{2} p^{2}+A_{1} p+A_{0}$,

$A_{3}=\frac{\left(h_{11}+h_{12}\right) h_{23}+\left(h_{13}+h_{14}\right) h_{21}}{h_{11} h_{14}-h_{12} h_{13}}$,

$A_{2}=\frac{-h_{14} h_{22}}{h_{11} h_{14}-h_{12} h_{13}}$

$A_{2}=\frac{-h_{22} h_{23}}{h_{11} h_{14}-h_{12} h_{13}}$,

$A_{0}=0$.

As part of the coefficients of this equation are negative, the system is unstable (which is obvious).

As the output signal of the system we take the sum of $\varphi$ and $\psi$, since these values are directly measured by the sensor, whereas the matrix of dependence of the output process $y$ and the vector of the state variable $x$ is of the form: $C=\left[\begin{array}{llll}0 & 1 & 0 & 1\end{array}\right]$.

We perform check of system controllability. The matrix of controllability will be the following:

$U=\left[\begin{array}{llll}B & A B & A^{2} B & A^{2} B\end{array}\right]$.

Since the object of control has a single channel, the criterion of its controllability is not zero determinant of this matrix: $\operatorname{det}(U) \neq 0$. It is easy to check by using the MATLAB software. In this case, the determinant of controllability is:

$\operatorname{det}(U)=\frac{\left(a_{2} a_{4} b^{2}+2 a_{3} a_{4} b^{2}\right)^{2}}{\left(a_{1} a_{3}-a_{2}^{2}\right)^{4}}$.

It is always positive, therefore, the object is controlled.

Let perform test of the observability of this system. Observability matrix has the form: 


$$
N=\left[\begin{array}{c}
C \\
C A \\
C A^{2} \\
C A^{3}
\end{array}\right] .
$$

This object is a single-channel, so it will be observed, if $\operatorname{det}(U) \neq 0$.

$$
\begin{aligned}
& \operatorname{det}(N)= \\
& =\frac{a_{2}^{2} a_{4}^{2}-2 a_{2} a_{3} a_{4}^{2}-4 a_{2} a_{4} a_{3}^{2}+a_{3}^{2} a_{4}^{2}+b a_{2} a_{4} a_{3}^{2}-4 a_{2} a_{4} a_{3}^{2}}{\left(a_{1} a_{3}-a_{2}^{2}\right)^{2}} .
\end{aligned}
$$

Therefore, the object is observed.

The modal synthesis method [2] was chosen to calculate the regulator. This method has been applied to solve the problem of stabilization for the following reasons. First, the basic mode of operation of the stabilization system of the robot is mode of initial conditions working off, and the modal method gives the best result in such systems. Second, to solve the problem of stabilization four state variables of the system must be evaluated, and two of them can be measured directly (tilt angle of the platform and the wheel rotation angle, respectively).

In accordance with the method of modal synthesis control law is as follows:

$u=K x, U=\left[\begin{array}{llll}k_{1} & k_{2} & k_{3} & k_{4}\end{array}\right]$.

Here $K$ is coefficient matrix of controller. Here the parameters of the matrix $K$ correspond to coefficient in the feedback under the following terms:

$k_{1}$ is derivative of the angle of the platform $\dot{\varphi}$,

$k_{2}$ is the angle of the platform $\varphi$,

$k_{3}$ is derivative wheel steering angle $\dot{\psi}$,

$k_{4}$ is angle of rotation of the wheel $\psi$,

Next, you must determine harakteristic equation of the system based on the following ratios:

$$
\begin{aligned}
& A(p)=\operatorname{det}(p l-(A+B K))= \\
& =p^{4}+A_{3}(K) p^{3}+A_{2}(K) p^{2}+A_{1}(K) p+A_{0} .
\end{aligned}
$$

After this the desired equation of the system must be proposed as following:

$$
C(p)=p^{4}+C_{3} p^{3}+C_{2} p^{2}+C_{1} p+C_{0} .
$$

To determine the coefficients of the controller (regulator) it is necessary to equate the coefficients of every power of the operator $p$ of characteristic equation to coefficients corresponding to ones of the desired equation.

\section{STRUCTURE OF ROBOT}

In accordance with the received matematical system model and the selected method of controller synthesis structure of the robot shown in Figure $\mathbf{3}$ was chosen.

\section{Sensor 1 STM32 Connection unit}

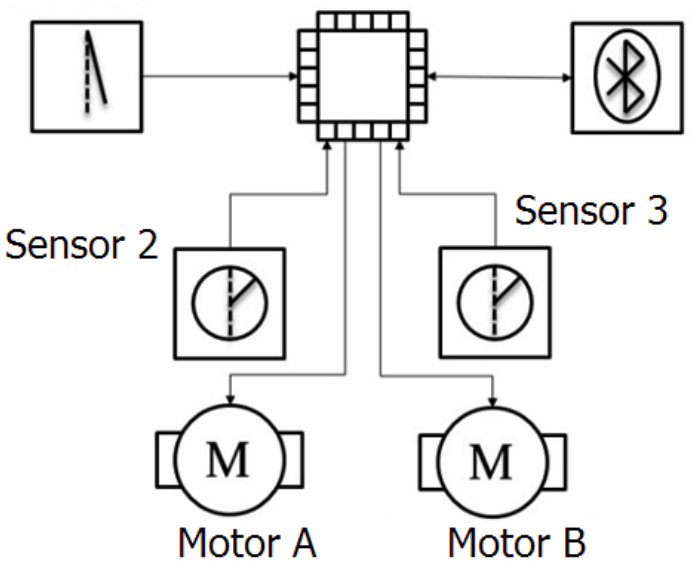

Figure 3: The block diagram of the robot.

The core of the system is a microcontroller STM32F100RBT6, mounted on the debug board STM32VLDISCOVERY. This microcontroller fully realizes the control of the system.

Measuring module MPU6050 applied as the inclination angle sensor. This module includes a triaxial accelerometer and a gyroscope. It may be used to determine not only the angle but also its first derivative (angular velocity) [3]. This allows you to directly measure another state variable, which significantly simplifies the process of developing of stabilization system.

As an actuator system servomotors NXT Lego Mindstorm were used. Encoders are integrated into the servomotors that allow us to determine the angle of rotation of the motor rotor relative to the stator. This means that it is possible to determine the angle of rotation of the wheel.

Also communication interface UART included in the module. For this module can be connected TTL-USB converter for communication with the $P C$ or the module Bluetooth, for example, HCO5. This module is introduced into the system to debug its operation, receiving the system parameters at the current time, and send commands to the device. 
Thus, we can formulate the following problems to be solved in the process of system development.

1. The implementation of servo control and data acquisition on the angle of rotation of the wheel.

2. Determination of the angle of inclination of the robot with a sensor module MPU6050.

3. Determination of the parameters of the mathematical model of the system.

4. Synthesis of control by modal method.

In the following sections of this paper the sequence of these tasks are described.

\section{ACTIVATION OF SERVOMOTOR}

One of the most common ways to control the motor voltage is pulse-width modulation. The input to the motor is supplied by rectangular pulses of constant frequency but different off-duty factor. At sufficiently high frequency, pulse voltage is smoothed by motor windings and is equivalent to a constant voltage a certain value.

For motor control timer built into the microcontroller was employed, which has four control channels configurable to their mode of generation of the PDM signal. Modulated voltage is supplied to the motor through a special chip, namely, voltage drivers.

\section{ESTIMATE OF ROTATION ANGLE AND THE WHEEL ROTATION SPEED}

To estimate the angle of rotation of the wheels builtin motor quadrature incremental encoders are used. Each encoder has two outputs: Channel $A$ and Channel B. For one turn of the wheel 180 pulses will be generated at both outputs. With all this, pulse time $T_{\mathrm{n}}$ is equal to pause time $T_{\Pi}$, which allows to measure the angle of rotation of the wheels with the accuracy up to one degree, by registration not only positive but negative fronts also. The signal on one channel is shifted relative to the other. It also allows you to define the direction of rotation of the wheel. Figure 4 is a diagram of a channel encoder signals when turning the wheel in a clockwise direction, and Figure $\mathbf{5}$ - when working counterclockwise.

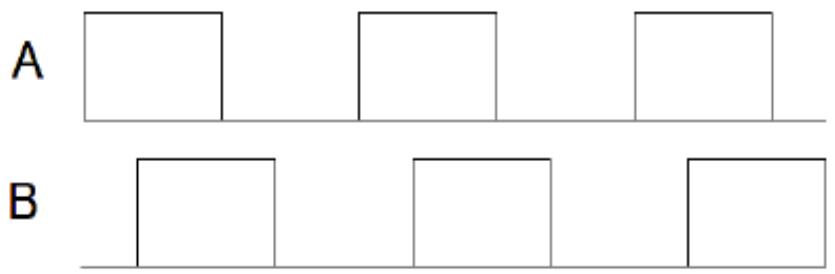

Figure 4: The encoder signal when turning clockwise.

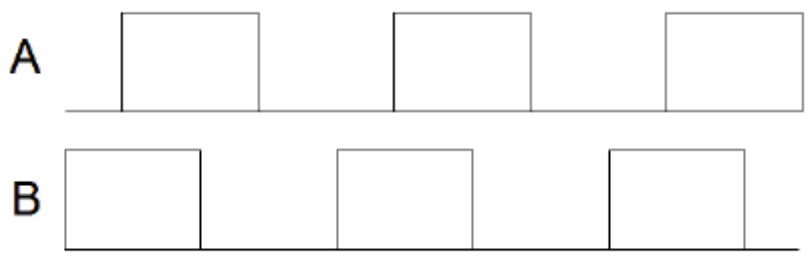

Figure 5: The encoder signals when turning the wheel anticlockwise.

The microcontroller timer was involved to register fronts. Each output of the encoder is connected to a separate channel of it. If registering a positive front on channel $A$ when channel $B$ value is zero or negative front on channel $A$ when channel $B$ value is unit, then the angle of rotation is incremented, in the opposite cases it is decremented.

To estimate the wheel speed it required to use a special filter called a differentiating filter.

Differentiating filter is a device that allows estimating the value to be filtered, as well as its derivatives. Consider the example of its work on the second order differentiating filter. The block diagram of the filter is shown in Figure 6.

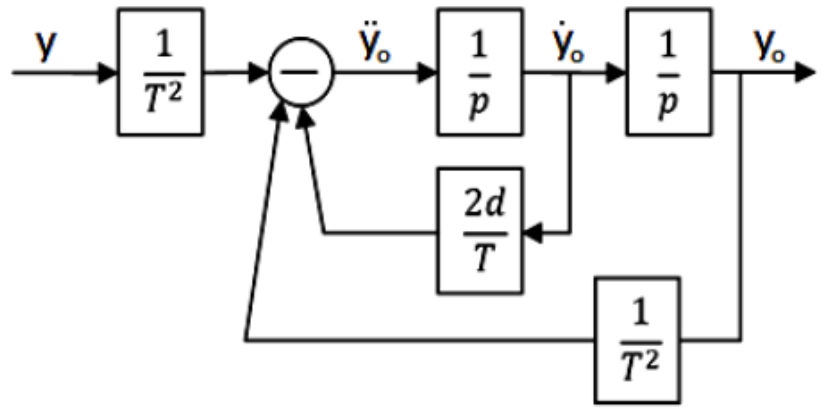

Figure 6: Block diagram of the second-order differentiating filter: $y$ is filtered signal, $y_{0}$ is its estimate, $\dot{y}_{0}=d y_{0} / d t$, $\ddot{y}_{0}=d \dot{y}_{0} / d t$.

This block diagram corresponds to the differential equation:

$y=T^{2} \ddot{y}_{0}+2 \xi T \dot{y}_{0}+y_{0}$.

The input filter is fed from the output of object, hence at its output we can obtain an estimate of the output signal of the object and its first derivative. Theoretically, this filter also provides an estimate of the second derivative (for this it is necessary to take a signal from the input of the first integrator), but due to noise of the output signal of the object the quality of obtained estimation of the second derivative is usually unsatisfactory. For the correct of determination of the $n$-th derivative it requires differentiating filter of order $n$ $+1[4]$. 
In the construction of the filter it is necessary to choose its right parameters. The main parameters of this filter is the filter time constant $T$ and the damping factor $d$. The first parameter specifies the duration of the transient processes in the filter, i.e., its speed. The second parameter specifies the oscillatory process in the filter. It is recommended to select this parameter within $0.5-1$. The value of the time constant of the filter is determined by the time constant of the processes in the object. For a correct evaluation of the output signal of the object it is necessary that the time constant of the filter was 5.3 times smaller than the smallest time constant of the object. However, one should remember that the smaller the time constant of the filter is, the worse is the effect of its using. Thus, the time constant must be small enough to delay the output signal of the filter was acceptable, but at the same time large enough to successfully smoothed the noises of the output signal.

The simplest way to implement the second-order differentiating filter with the structure described above, is to replace the integration operation by the summation operations:

$y=\int_{t 1}^{t 2} \dot{y} d t \Rightarrow y[k]=y[k-1]+\Delta y \times \Delta t$.

Here $y[k-1]$ is filtered parameter value in the previous step, $y[k]$ is the current value, $\Delta y[k]$ is the input parameter of the integrator (the derivative of the output signal) at the current step, $\Delta t$ is integration step. Second order filter can be implemented in a consistent implementation of the following operations:

$\Delta^{2} \hat{y}[k]=\frac{y[k]}{T^{2}}-\frac{2 \xi \cdot \Delta \hat{y}[k-1]}{T}-\frac{\Delta \hat{y}[k-1]}{T^{2}}$

$\Delta \hat{y}[k]=\Delta \hat{y}[k-1]+\Delta^{2} \hat{y}[k] \Delta t$,

$\hat{y}[k]=\hat{y}[k-1]+\Delta \hat{y}[k] \Delta t$.

Here $\Delta^{2} \hat{y}[k]$ is the estimate of the second derivative, $\Delta \hat{y}$ is evaluation of value of the first derivative, $\hat{y}$ is the estimate of the value of the filtered quantity, $y$ is the value of the filtered value.

In contrast to the theoretical model of the filter, in the implemented on the microcontroller filter there is another important parameter that needs to be choosen correctly. This is the integration step $\Delta t$. The basic rule in this choice is that its value must be smaller than the smallest filter parameter. For correct operation of the filter it is necessary that the integration step was less than the time constant at least in 3 times.

There is another feature of the implementation of the filter on the base of microcontroller. Most microcontrollers can operate with only integer arithmetic valuse, therefore, to avoid data loss when divided, you must perform the left shift a few bits before the operation of the division (which is equivalent to multiplying by 2 to some extent), and after the completion of all operations the reverse shift must be performed, which is equivalent to division.

For above operations in real-time regime, it may be implemented as a function that is called after intervals equal to the selected integration step. The results of the differentiating filter working applied to estimate the wheel speed are shown below. Figure 7 shows the results of measuring the angle of rotation of the wheel, and Figure 8 shows the results of the wheel speed according to the steering angle.

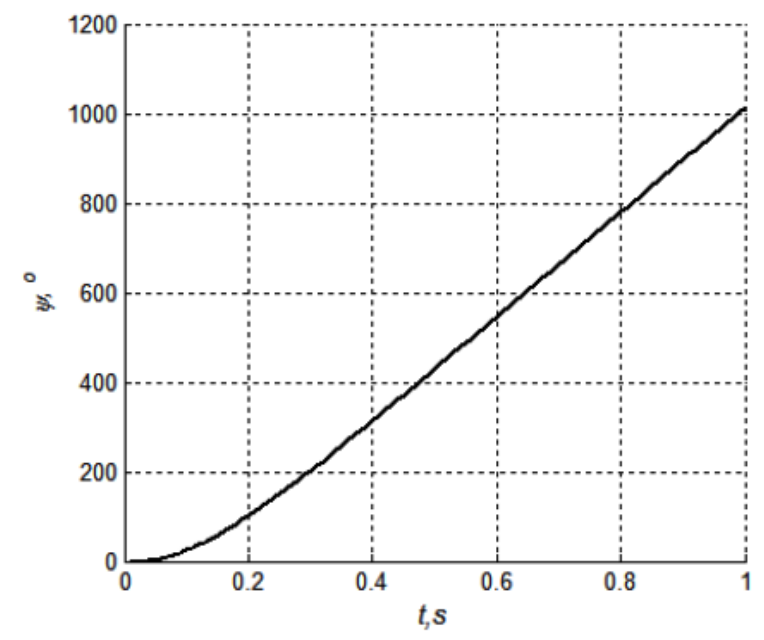

Figure 7: The angle of rotation of the wheel.

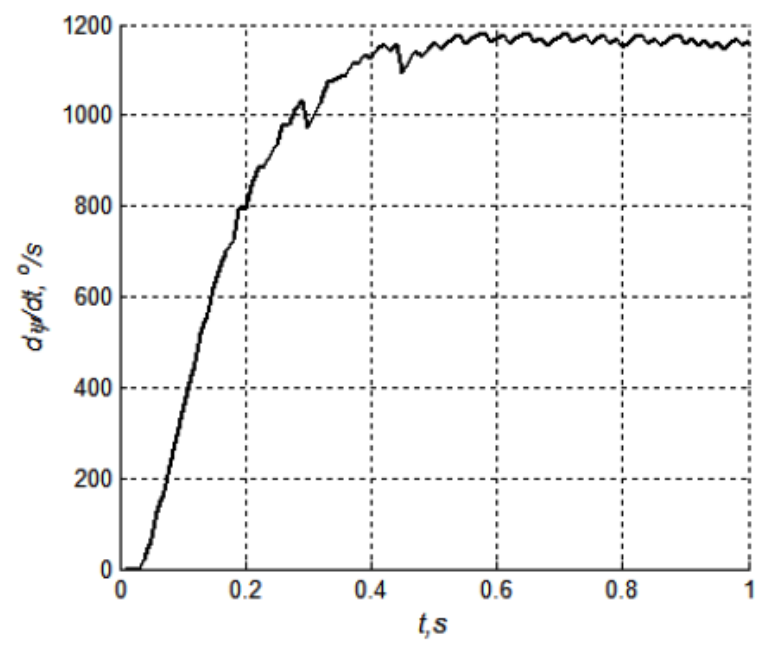

Figure 8: The estimate of the wheel rotatopn speed.

\section{GETTING OF MATHEMATICAL MODEL OF THE SERVOMOTOR}

To operate the motor in accordance with the control algorithm of the system it is necessary to obtain a 
mathematical model of it. Approximately behavior of the DC motor is described by the following relationships:

$J \varepsilon=M-M_{1} ; M=k_{m} I$

$U-E=I R ; E=k_{e} I$

$\frac{k_{m}}{R} U=J \dot{\omega}+\frac{k_{m} k_{e}}{R} \omega+M_{1}$,

Here $J$ is moment of inertia of the shaft; $M$ is the moment produced by the motor; $M_{1}$ is load moment; $k_{\mathrm{m}}$ and $k_{\mathrm{e}}$ are structural factors of the motor; $R$ is resistance of the armature winding; $E$ is backward EMF.

At idling of motor with voltage $U_{0}$ at the input of motor, solution of the differential equation (15) is a following function:

$\omega(t)=U_{0} K\left(1-e^{-t / T}\right)$.

Here $K=1 / k_{e}, T=J R /\left(k_{m} k_{e}\right)$. If we integrate this equation over the interval from 0 to $t$, we obtain the expression for the angle of rotation of the motor rotor:

$\varphi(t)=\int_{0}^{t} \omega(t) d t=U_{0} K(t-T)+U_{0} K T e^{-t / T}$.

As seen from the equation (17), with time the angle of rotation approaches the line described by the equation:

$\varphi(t)=U_{0} K(t-T)$.

For mathematical motor model, measurements were made of the angle of rotation of the rotor at a voltage $U=5 \mathrm{~V}$. The results of measurement of the angle of rotation of the motor are shown in Figure 9. Points at the linear part of the graph are approximated by a straight line, the coefficients of which were used to determine parameters of the model:

$K=1,56 \mathrm{~V} \cdot \mathrm{s} / \mathrm{rad}, T=0,12 \mathrm{~s}$.

To test this model the system was simulated with the obtained values of the parameters. Figure $\mathbf{1 0}$ shows the results of modeling of the systems and data obtained from the results of measurement for the speed of rotation of the motor.

Next, it is nessesary to determine the constructive coefficients of the motor $k_{\mathrm{m}}$ and $k_{\mathrm{e}}$. The value $k_{\mathrm{e}}$ is completely determined by the parameter $K$, namely: $K$ $=1 / k_{\mathrm{e}}$.

However, this paraaameter has significant non- linearity. The reasons of non-linearity are the design features of the motor, as well as driver microcirqites used for motor control. Figure 11 shows the transient responses of the motor at input voltages from $1 V$ to 10 $V$ with steps of $1 \mathrm{~V}$. Figure 12 shows dependence of the parameter $k_{\mathrm{e}}$ from the input voltage.

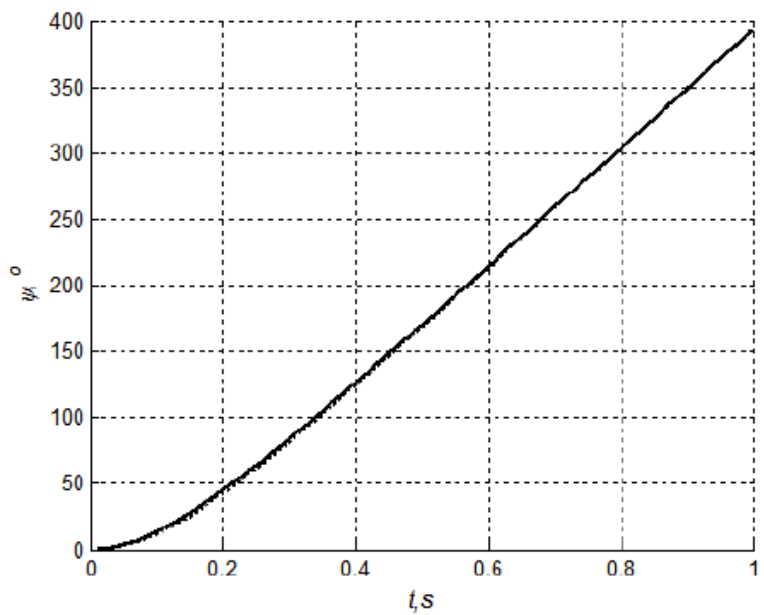

Figure 9: Data for the angle of rotation of the motor rotor: the first (solid) curve is the experimental data, the second (dotted) curve is the data on the results of the simulation; both curves flowed together and became inseparable.

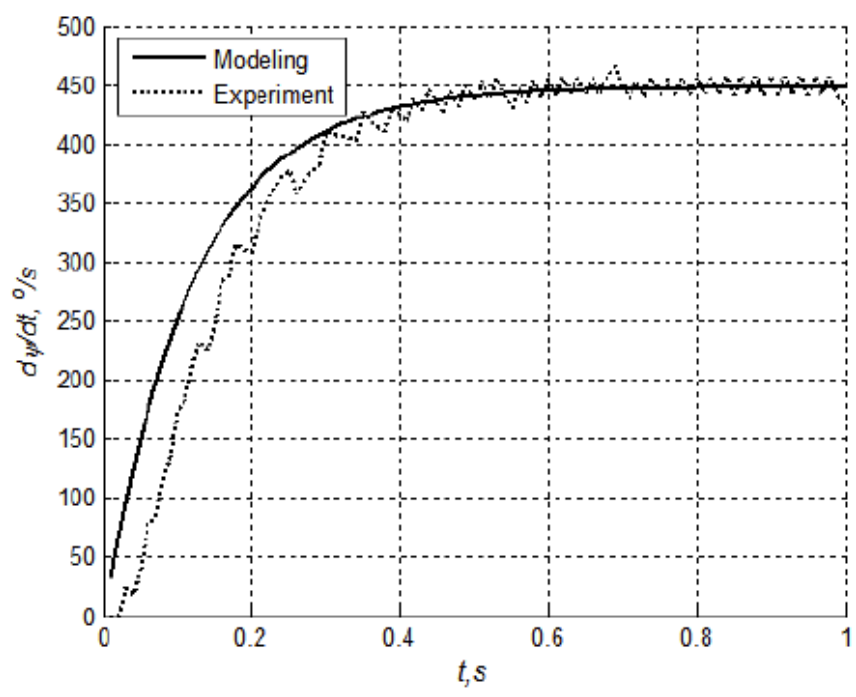

Figure 10: Data for the rotational speed of the rotor of the motor: the doted curve is the experimental data; the solid curve is the data on the results of the simulation.

To determine the parameter $k_{\mathrm{m}}$ experiment was conducted, during which the value of inrush current was measured with known load mometn on the motor shaft. The experimental setup is shown at Figure 13.

On the wheel connected to the motor shaft the load was fastened to via the thread. First the force of pressure of the load on the support was measured when the motor is switched off. Then voltage was applied to the motor, wherein the rotor current is 


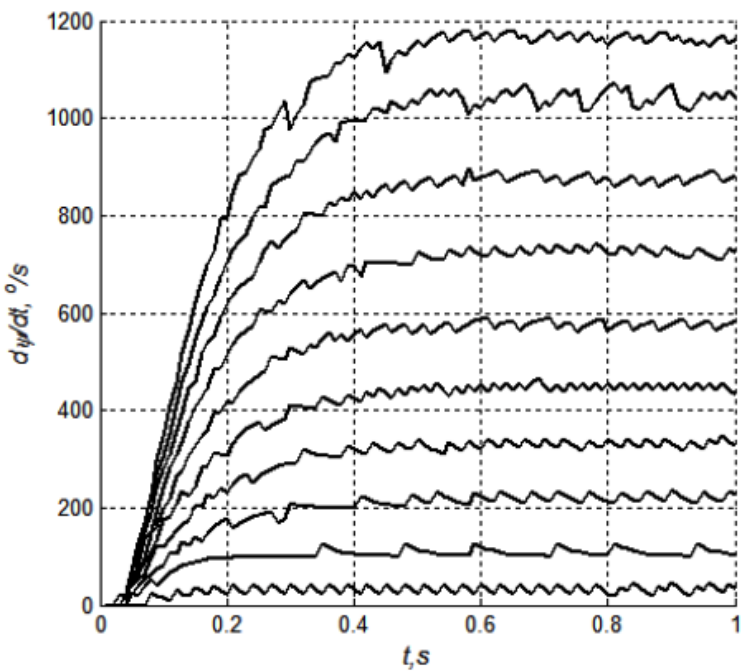

Figure 11: Transitional characteristics of the motor with different input voltages.

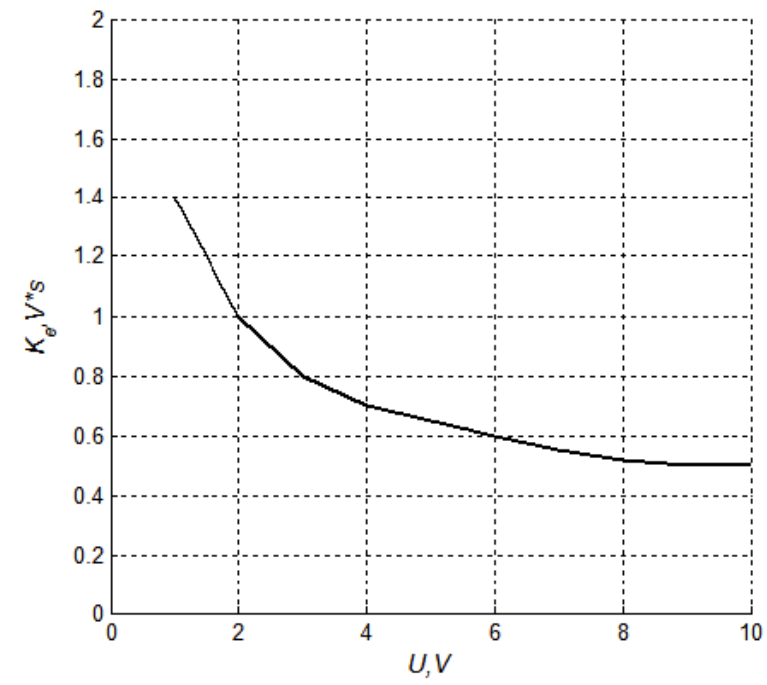

Figure 12: The dependence of the parameters $k_{e}$ on the input voltage.

measured. The force of the load pressure was reduced by the force of the thread tension. Knowing the strength and the radius of the wheel, it is easy to determine the moment generated by the wheel:

$M_{\kappa}=r \cdot F_{H}=r\left(F_{\Gamma 1}-F_{\Gamma 2}\right)$,

Here $F_{\Gamma 1}$ is pressure force to support the load when the motor is switched off; $F_{\ulcorner 2}$ is the same when motor is enabled. Figure 14 shows the resulting dependence of the moment of the wheels on the current in the rotor circuit.

The value $k_{m}$ was determined from the expression:

$k_{m}=M / I$.

On the basis of the experiment the following coefficient was determined: $k_{\mathrm{m}}=0.25$.

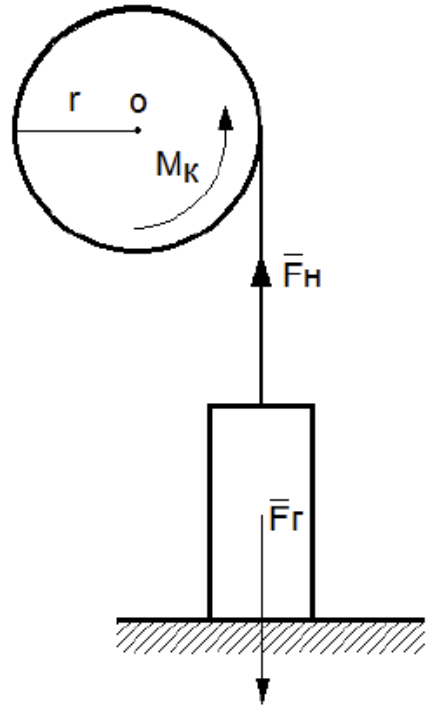

Figure 13: The scheme of the experiment to determine the structural parameter $k_{m} . M_{k}$ is the moment produced by the wheel; $O$ is wheel axle; $r$ is the radius of the wheel; $\bar{F}_{H}$ is the thread tension force (vector); $\bar{F}_{\Gamma}$ is the power of the pressure of the load on the support (vector).

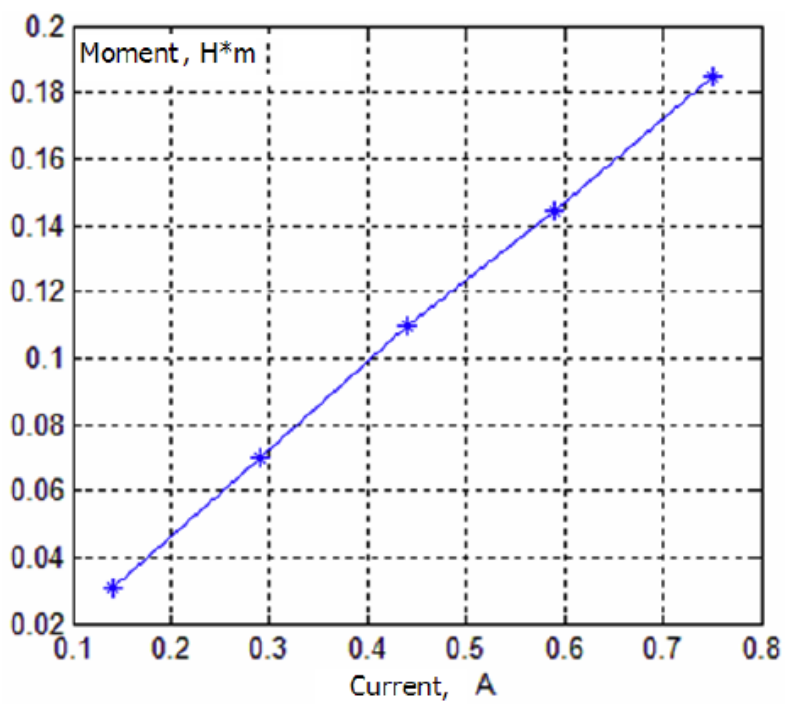

Figure 14: The dependence of the moment of the wheel on the current in the rotor circuit.

Woth known values of the parameters $k_{\mathrm{m}}$ and $k_{\mathrm{e}}$, and the resistance of the anchor chain $R$, which may be determined with a multimeter, we can calculate the value of the moment of inertia $J$. Since the motor is integrated into the motor gearbox, inertia value, obtained according to the procedure described above, will match the output of the gearbox.

\section{PRINCIPLE OF ACCELEROMETER WORKING}

Accelerometer is a device that measures the projection on its axis of its acceleration (hence, sum of all forces applied to its case, except gravity one) [3]. 
That is, it measures the projection of the apparent acceleration (the difference between the true acceleration of the object and the gravitational acceleration). It follows that the use of the accelerometer for determining the angle of inclination is useful when the force applied to its case (reaction forces) are equal in magnitude to the force of gravity and it directed in the opposite direction, that is, the sensor should be at rest, or at least it can move without acceleration in real systems is not common.

There are several types of accelerometers, differing in the sensing elements and in the principle of action. Figure 15 is a block diagram of single-axis accelerometer, which converts the change in the position sensor 2 into a voltage via potentiometer 3 .

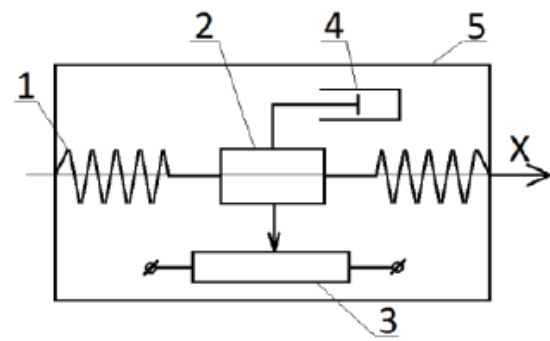

Figure 15: Internal structure of a uniaxial accelerometer; 1 is spring, 2 is sensor, 3 is potentiometer, 4 is damper, 5 is the case.

The sensing element is a weight secured to springs that are attached to the case. The damper is used to reduce the impact of natural oscillations of the sensing element. At Figure 15 no force is applied to the body along the axis $X$. If the acceleration of the case takes place, the result will change; it will be as shown in Figure 16

At that, force $F 1$ acts to the case, the sensing element moves to the left, in the opposite direction relatively the diration of the force. The potentiometer registers this shift and voltage appears at the sensor output, which is proportional to the applied acceleration (and force).

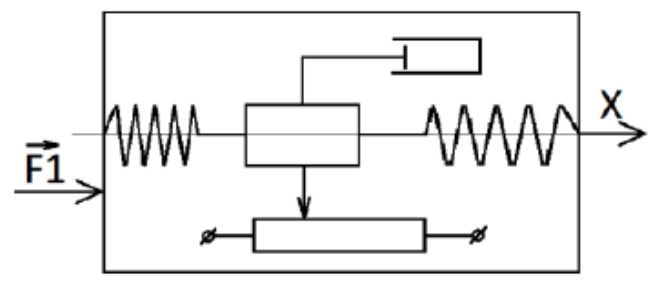

Figure 16: Internal structure of uniaxial accelerometer when subjected to forces along its axis.

If the single-axis accelerometer positioned perpendicular to the earth's surface, that is, along the gravity vector, the sensor will make the measurement of ground reaction forces, as shown in Figure 17.

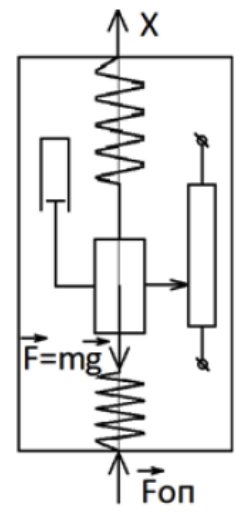

Figure 17: Internal device of the uniaxial accelerometer when measured reaction force.

By turning the sensor from the position in Figure 17 to the position in Figure 18, the projection of force $F$ on the $X$-axis will decrease. You get a two-axis accelerometer if add the same second accelerometer to this uniaxial one. It should be positioned with its axis transversely to the first axis.

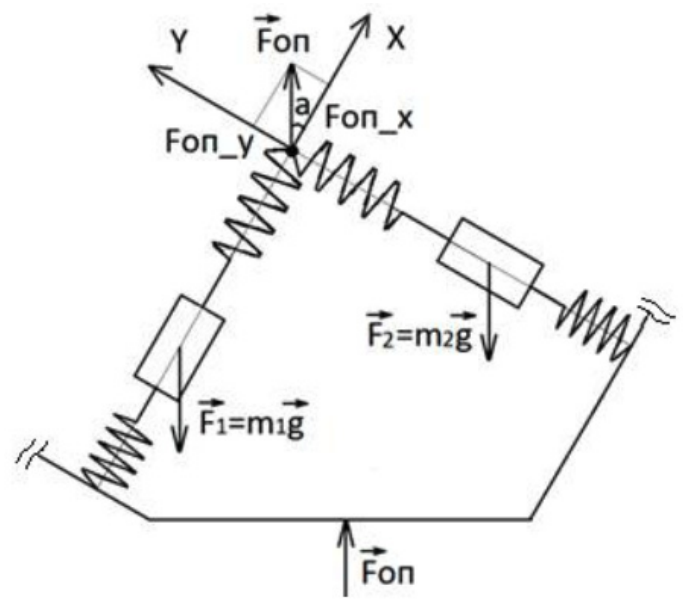

Figure 18: The settlement scheme of angle of the vector projections reaction forces on the axis of the accelerometer.

As is known, with the projections of the vector on the two axes (OX, OY) of the Cartesian coordinate system, the angle of the vector can be restored.

\section{PRINCIPLE OF GYRO WORKING}

There are several types of MEMS gyroscopes, differing in its internal device, but they all are common in their work based on the Coriolis force. Each of them has a working body, it makes reciprocating motion. If rotate the substrate on which the body is, then Coriolis force will start to operate. It is directed perpendicularly to the axis of rotation and to the direction of motion of the body. Figure 19 shows the mechanism of this force.

Knowing the linear velocity and the Coriolis force we can determine angular velocity. 


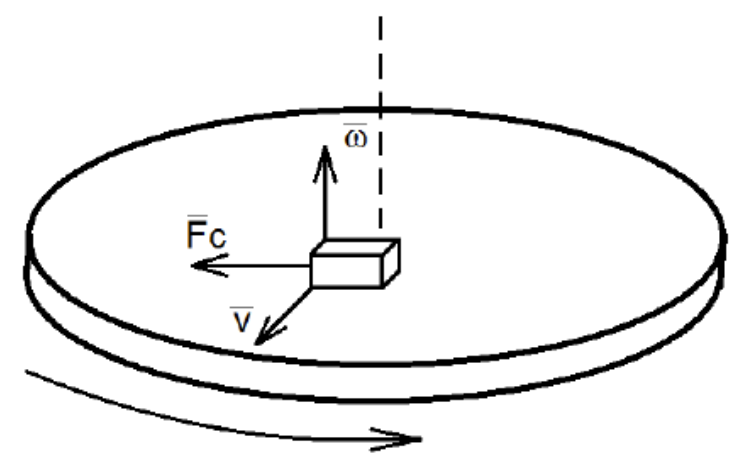

Figure 19: The mechanism of the Coriolis force: $\bar{\omega}$ - angular velocity; $\bar{v}$ - the vector of linear speed; $\bar{F}_{C}$ - the Coriolis force.

One of the possible implementations of the gyroscope has the following structure: fixed on flexible suspensions frame inside which some weight makes reciprocating oscillatory motion [4]. The structure of this sensor is shown in Figure 20.

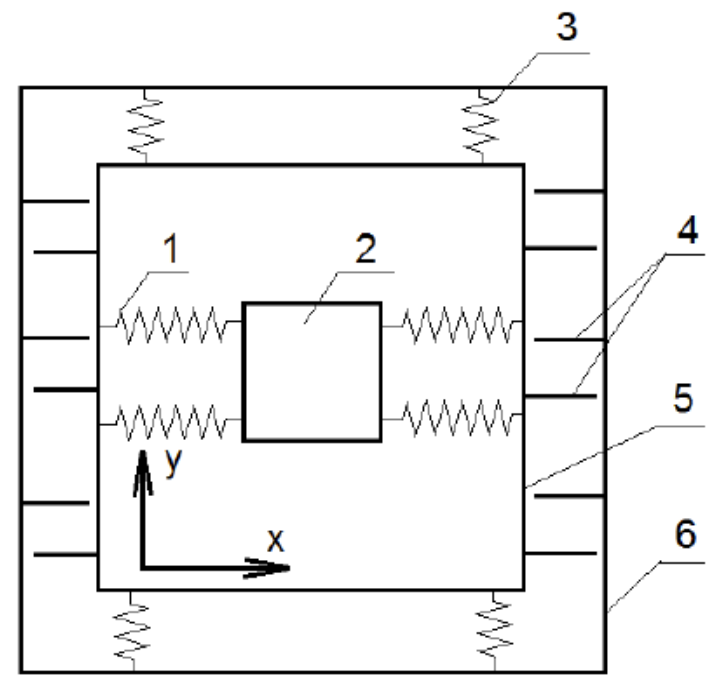

Figure 20: The internal structure of the gyroscope: 1 fastening of the mass; 2 - operating weight; 3 - fastening the inner frame; 4 - sensor of displacement of the inner frame; 5 internal frame; 6 - substrate.

Oscillations of working mass occur along the $X$ axis and are electrostatically generated. The vibrations of the inner frame is possible only along the axis $Y$. Capacitor plates (displacement sensors) are disposed between the inner frame and substrate. It allows measuring of the capacity, it is possible to sence the frame movements relatively the substrate.

Figure 21 shows the structure of the gyroscope during its rotation in the $X Y$-plane clockwise.

The fluctuations in the inner frame can be caused not only by the Coriolis force, but also by the linear accelerations which act along the axis $Y$. The problem is solved by placing the substrate in one of two frames, each of which contains the working mass. Both masses oscillate in antiphase, hence at some given time, the Coriolis force acting on the first mass, is directed opposite to the force acting on the second one. The signals generated by the Coriolis force will be sumed, and the in-phase component generated by the linear acceleration will be deducted.

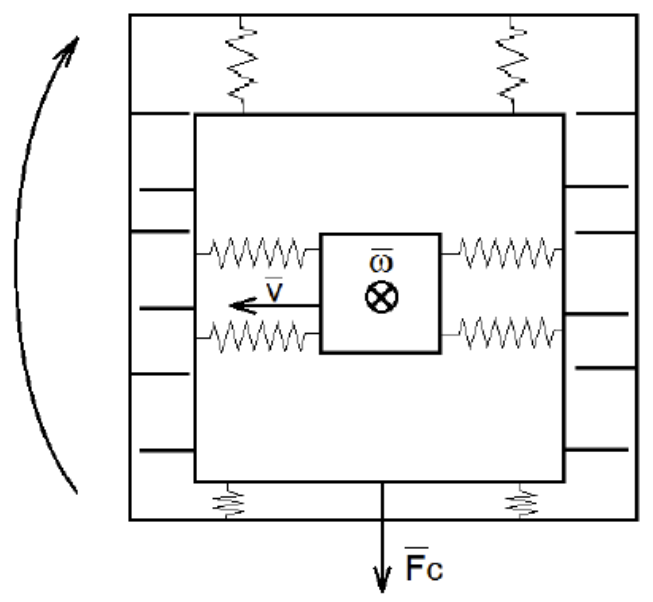

Figure 21: The structure of the gyroscope in rotation: $\bar{\omega}-$ the angular velocity vector; $\bar{v}-$ the vector of linear speed; $\bar{F}_{C}-$ the Coriolis force.

Thus, by measuring of the deflection of the inner frame by means of shift sensors we can estimate the angular velocity of the sensor.

\section{CONNECTION OF MPU6050}

As the tilt measuring sensor of the robot module MPU6050 is used. This module includes two MEMS sensor: accelerometer and gyroscope. The module has a digital output $I^{2} C$. Integrated $A D C$ converts analog sensor signals into digital code for transmission it over the interface $I^{2} \mathrm{C}$. Below are the technical specifications of the module.

- $\quad$ Three-axis gyroscope;

- Three-axis accelerometer;

- $\quad$ Temperature sensor;

- $\quad$ Feeding voltage from $2.375 \mathrm{~V}$ to $3.46 \mathrm{~V}$;

- $\quad$ FIFO-buffer with capacity of 1024 bytes;

- User programmable digital filters for the gyroscope, accelerometer and temperature sensor;

- $\quad \quad^{2} C$ interface for reading and writing of the device registers, clocked at up to $400 \mathrm{kHz}$;

- User programmable measuring range: $\pm 250, \pm$ $500, \pm 1000$ and $\pm 2000 \%$; 
- $\quad$ Built-in 16-bit $A D C$;

- $\quad$ Digital programmable low pass filter;

- $\quad$ The current in mode of operation is $3.6 \mathrm{~mA}$;

- $\quad$ Standby current is $5 \mu A$.

MPU6050 has been connected to the $I^{2} C$ module of the mikrocontroller STM32F100RBT6B for sending of commands and reading of registers of the required data. After sending to the module of the measurement start command, the regular digitization of readings from all axes gyroscope, accelerometer and temperature sensor begins. One should only read information from the necessary registers. The frequency of new data loading into these registers by ADC is dependent on the choised by the user's sensitivity of the sensor, and hence on the choiced measuring range.

\section{DETERMINATION OF THE ANGLE DEVIATION OF THE VERTICAL LINE}

Angle measurement signals obtained by the accelerometer contain high frequency noise even in static mode. In the dynamics, the linear accelerations lead to the fact that the measurements of the angle can be even incorrect. The first problem can be solved by filtering, but this leads to a phase shift of the useful signal, which can make the system unstable. Many objects of control can suppress the high-frequency component of its inertness, and smoothing can be not nesessary, but the second problem with the incorrect determination of the deflection angle of the accelerometer when subjected to a linear acceleration, can easily make the system unstable, even when correctly calculated regulator.

Measuring the angle of a gyroscope gives rise to low-frequency noise that occurs because of integrating the angular rate (because gyro measures namely this value). This result in drift of zero point and the angle will progressively increase or decrease, even if the system is steady.

The accelerometer measures the projection on its axis of the sum of all the acceleration (connected with the forces) applied to it, except for gravity. That is, in a static reaction force on the device is measured. Since this force is opposite to the direction of gravity, then knowing magnitude of its projections on the axes, it is possible to determine the deflection angle from vertical line. This can be done using the formula:

$\varphi_{A}[i+1]=\operatorname{arctg}\left(\frac{F_{\text {on_y }_{-}}[i+1]}{F_{\text {on_x }_{-}}[i+1]}\right)$,

Here $\varphi_{A \kappa C}[i+1]$ is the deflection angle at the current time calculated via accelerometer: $F_{o n_{-} y}[i+1]$ is projection of reaction force onto the axis $Y$, and $F_{\text {on } x}[i+1]$ it the same on the axis $X$.

The gyroscope measures an angular speed in a specific plane using the Coriolis Effect. In this case we are interested in the angular velocity in the plane $X O Y$. To get the angle we can integrate gyroscope signal:

$\varphi_{H}(t)=\int_{0}^{t} \omega(\tau) d \tau$

When implemented on a microcontroller, this operation is replaced by numerical integration:

$$
\begin{aligned}
& \varphi_{H}[i+1]=\varphi_{H}[i]+\Delta \varphi_{H}[i+1]= \\
& =\varphi_{H}[i]+\omega[i] \Delta t .
\end{aligned}
$$

Here $\varphi_{H}[i+1], \varphi_{H}[i]$ is angle calculated using the gyroscope in the current and previous step; $\omega[i]$ is the angular velocity of the previous step; $\Delta \varphi_{H}[i+1]$ is angle increment in the interval of time equal to the integration step; $\Delta t$ is step of integration.

Figures 22 and $\mathbf{2 3}$ are graphs of angles obtained using the gyro and accelerometer data included into measuring system MPU6050.

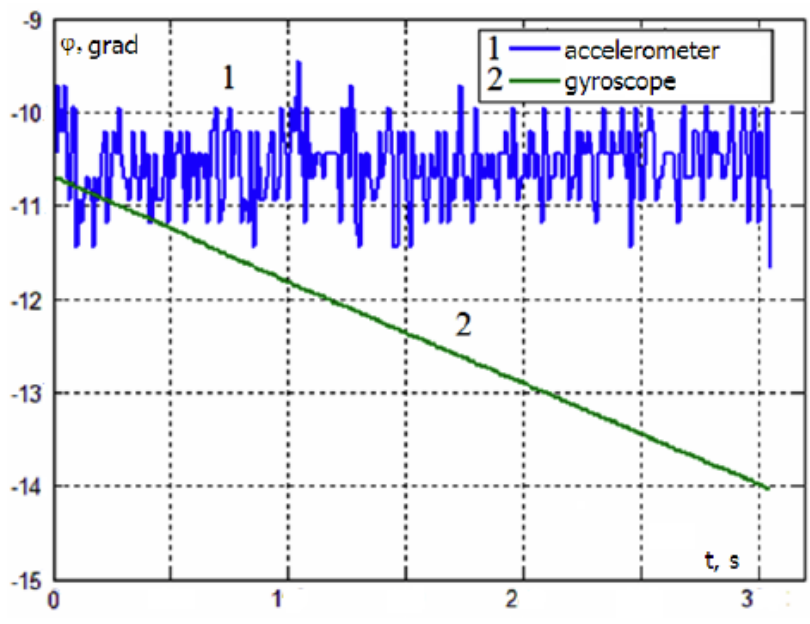

Figure 22: The angle calculated using the accelerometer and gyroscope in statics.

Since both of these sensors have disadvantages they cannot be used directly in applications where high accuracy of estimation of the angle of inclination is necessary. Special filter is nesessary which would use the advantages of each sensor and compensate disadvantages. One of the possible embodiments of such a filter is a complementary filter, or alpha-beta filter.

For convenience, the initial angle of the gyroscope is chosen as close as possible to the corner of the 
accelerometer. From Figure $\mathbf{2 2}$ it is clear that variations in the angle calculated from the accelerometer readings are greater than one degree, and Figure $\mathbf{2 3}$ demonstrates four degree error in its determination in the dynamics. Indications of the angle calculated from angular rate gyroscope, a correct on the second chart, but on the first ont there is a significant drift of this angle.

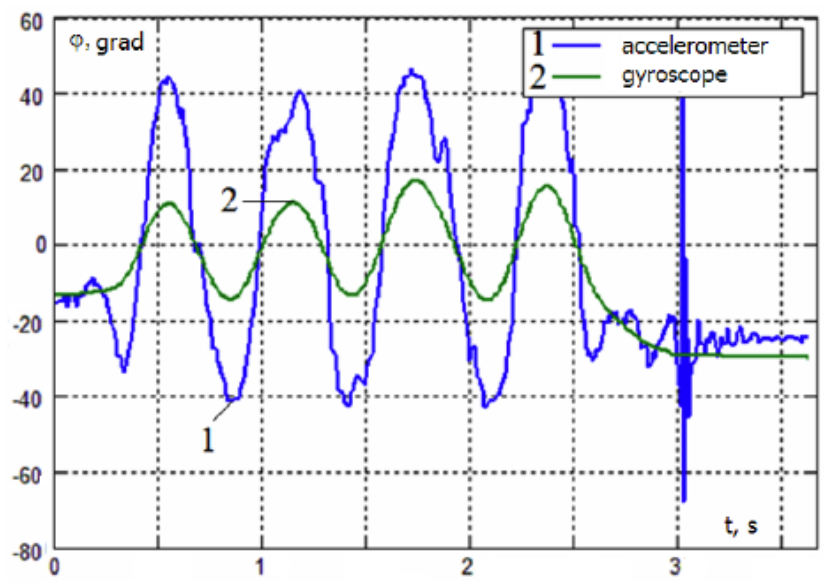

Figure 23: The angle calculated using the accelerometer and gyroscope in dynamics.

\section{THE COMPLEMENTARY FILTERS}

Complementary filter or alpha-beta filter in this case is used to determine the angle and operates with the output signals of accelerometers and gyroscopes. Below is the formula for the calculation of this angle:

$\varphi[i+1]=(1-K)\left(\varphi[i]+\Delta \varphi_{H}[i+1]\right)+$

$+K \varphi_{A}[i+1]$

Here $K$ is filter coefficient, which value is between 0 and $1, \varphi[i], \varphi[i+1]$ are the angles calculated by the filter to the current and previous steps.

Substituting (17) and (18) into (19), we obtain the final expression for the calculation of the angle of deflection:

$\varphi[i+1]=(1-K)(\varphi[i]+\omega[i] \Delta t)+$

$+K \cdot \operatorname{arctg}\left(\frac{F_{\text {on_y }_{-}}[i+1]}{F_{\text {on_x }}[i+1]}\right)$.

This filter is a simplified version of the Kalman filter for the one-dimensional case, where the first term is a unit of prediction. At thet the angle increment is determined not on the basis of a mathematical model of the system and the known values of control signal, but from the angular velocity at the previous step, which is measured directly by the gyroscope. In addition, the coefficient $K$ is a constant. All this greatly simplifies the calculation process, and the resulting angle can be considered as sufficiently accurate approximation to the real value. The use of the gyroscope is good because we get on its output the value of the angular velocity, which saves us from having to differentiate the value of the angle that has a high-frequency component. It is particularly important that its results are not affected by the linear acceleration. Therefore, during the designing of the control algorithm, it is desirable if possible the state variables were measured by the sensor, rather than calculated from the values of other, related, values. This will positively affect the stability margin and quality control.

By changing the value of the coefficient $K$, we define wich of the terms will have greater weight. With decreasing of $K$ filter readings will tend to readings of the gyroscope, with the increasing it will tend to ones of the accelerometer. When $K=0$, the equation (19) degenerates into (18), and we get the angle without high-frequency noises, but floating away. When $K=1$, equation (19) degenerates to (17), and the computed angle is very noisy and it is affected by linear acceleration. The task is to correct selection of this ratio to estimate the angle obtained from the output of the filter meet the specified requirements. Figures 24 and 25 show graphs for different values of the parameter $K$. Step of sampling is $1 \mathrm{~ms}$. The initial value of the angle of the gyroscope and the filter are set different from the initial value of the angle of the accelerometer. It differs by 2 degrees for easy assessment of slow processes in the filter. In a running system, these values must be the same as shown in Figure 26.

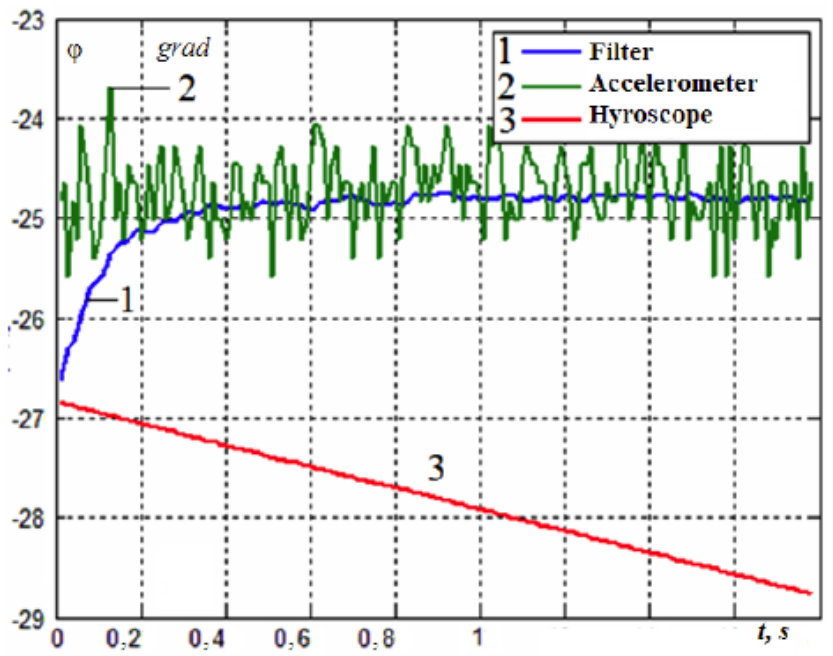

Figure 24: The angles of the gyroscope, accelerometer, and alpha-beta filter when $K=0.01$.

As can be seen from the graphs, with a decrease in the value of the parameter $K$ the flow time of slow 
processes in the filter increases. This is due to the weakening of the influence of the data of accelerometer (line became smoother) and the impact of the gyroscope increase. The change of the initial conditions does not affect to the time of developing of slow processes (see Figures 25 and 26). You can also observe the static error, which will grow with decreasing coefficient $K$ and increase in the rate of the gyro bias angle, but this rate can be considered constant. It depends on the sensor accuracy and computational error: the smaller is step of integration, the smaller is the error and slower drift. The static error does not depend on the initial conditions. When $K=$ 0.001 , it was about $1.2^{\circ}$. For the most accurate operation of the initial value of the filter is recommended to equate to the initial value of the angle of the accelerometer. The displacement can be calculated empirically, and it will be the same throughout the range of the angle measurement.

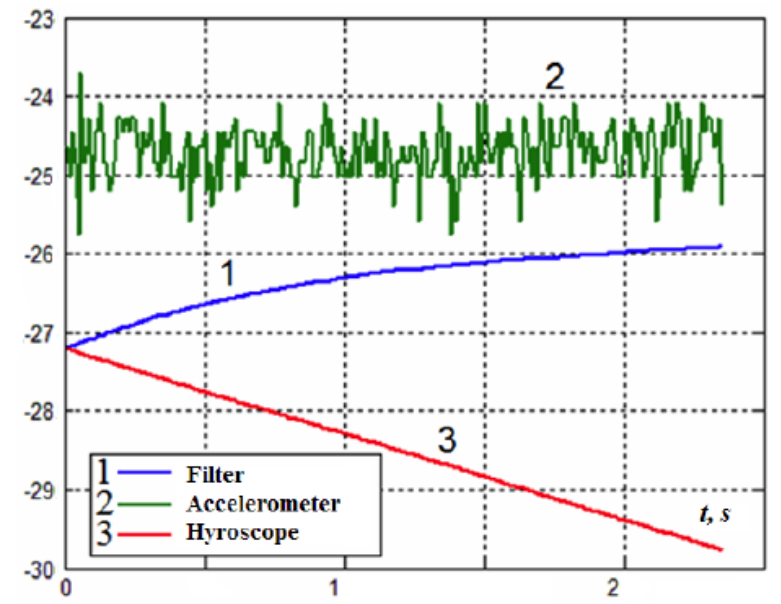

Figure 25: The angles of the gyroscope, accelerometer, and alpha-beta filter when $K=0.001$.

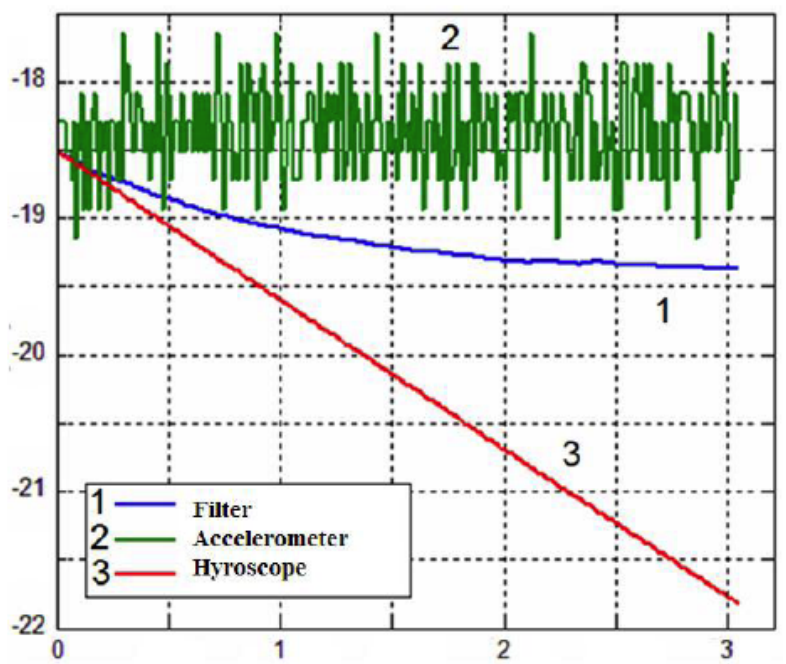

Figure 26: The angles of the gyroscope, accelerometer, and alpha-beta filter when $K=0.001$, and the same initial conditions.
Figures 27 and 28 are graphs of angles of gyroscope, accelerometer, and alpha-beta filter in dynamics for different values of the parameter $K$. In Figure 27 angle of the filter mostly corresponds to the angle of the gyroscope. This means that for the coefficient $K$ the best value is 0.001 , in spite of the longer time of slow processes. Measuring the displacement, we can compensate for it, but over the course of time the slow processes (the first three seconds) angle will be not accurately: the largest value of the error is at zero time and is equal to the offset value. Nevertheless, a mistake about $1-2^{\circ}$ regulator will work off more successfully than the errors introduced by the accelerometer for large values of $K$. In dynamics delay of the filter is minimal (with $K=$ 0.001 it is less than $20 \mathrm{~ms}$ ).

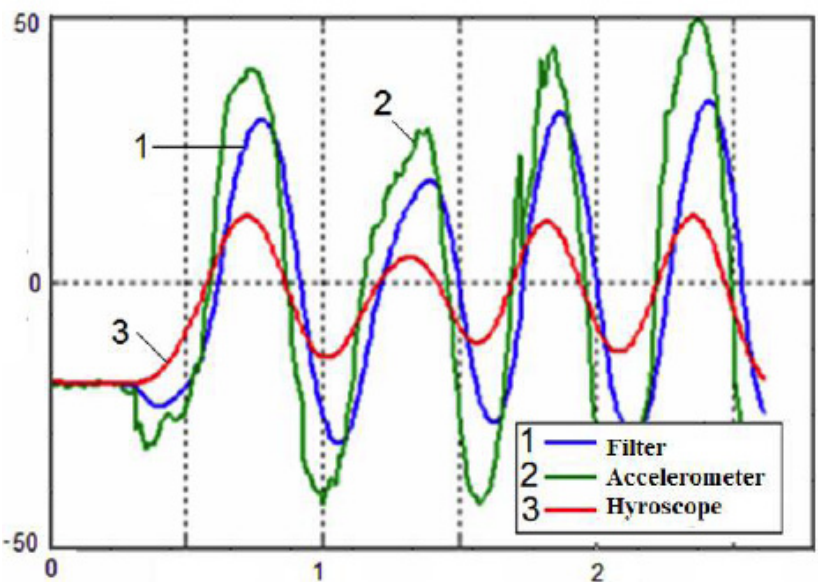

Figure 27: The angles of the gyroscope, accelerometer, and alpha-beta filter in the dynamics when $K=0.01$.

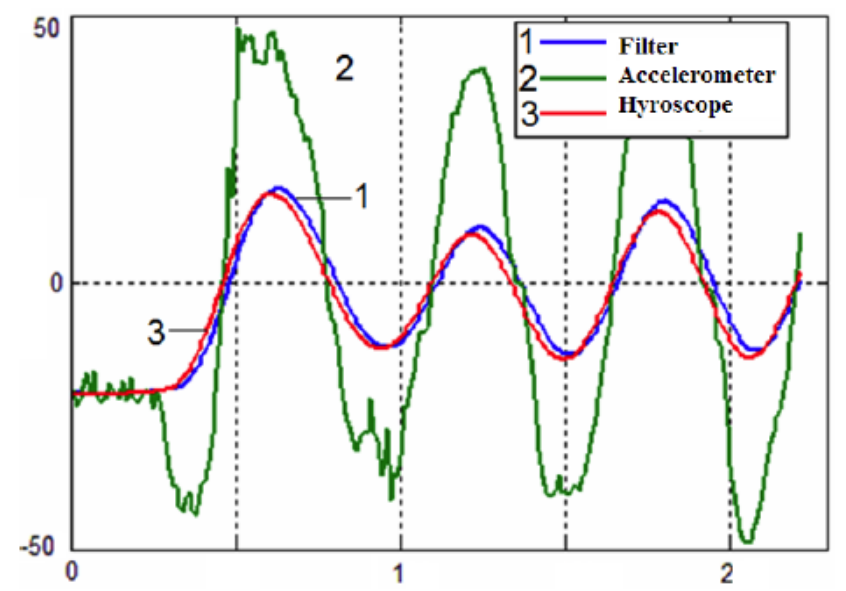

Figure 28: The angles of the gyroscope, accelerometer, and an alpha-beta filter in the dynamics when $K=0.001$.

Figure 29 shows plots of angles when exposed linear acceleration along an axis parallel to the surface on which the robot will move the balancing. This acceleration can occur during acceleration and braking. The graphs show that they have little impact on the 
value of the estimated angle even with ten times increased error of the accelerometer.

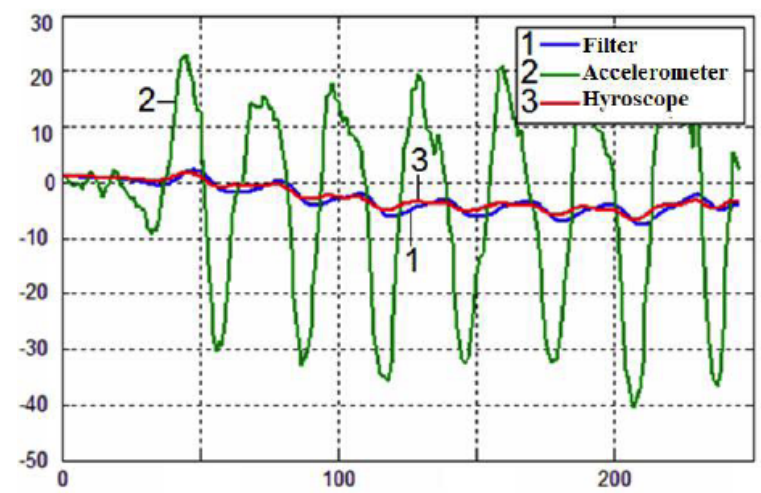

Figure 29: Angles when linear accelerations exposed.

Figure $\mathbf{3 0}$ shows graphs of changing of angles at fall of balancing robot, which initially was in upright position. The graphs show that the fall of the robot in one direction accelerometer initially identified as falling into another due to its internal structure. In a system with feedback, it may result in the appearance of a positive feedback, which can make the system unstable. However, the use of the complementary filter solves this problem.

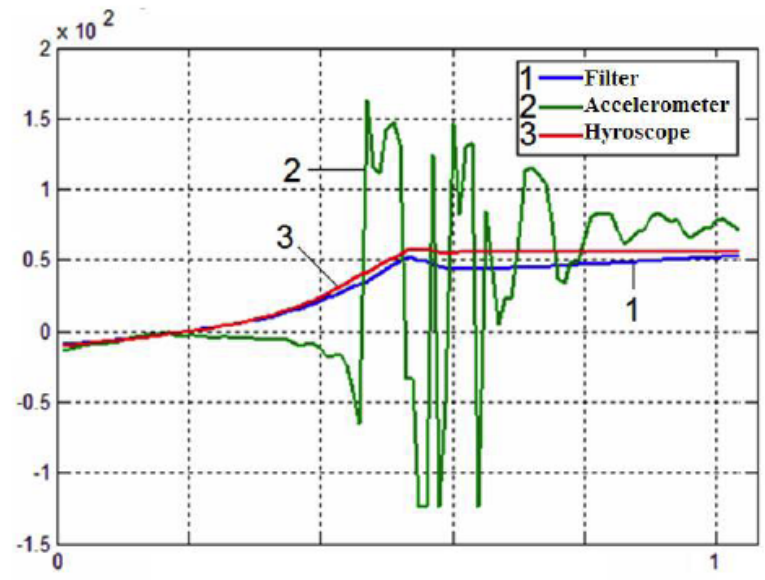

Figure 30: Angles when the robot fall from the vertical position.

\section{DEFINITION OF THE MATHEMATICAL MODEL PARAMETERS}

Before calculation of the regulator, it is necessary to determine the remaining unknown parameters of a mathematical model of the system. These parameters are:

1. The weight of the platform.

2. The distance from the center of mass of the platform to the suspension point of the pendulum (I).

3. The radius of gyration platform $(\lambda)$.
Mass robot was determined using an electronic scale and it is equal $m=0.52 \mathrm{~kg}$.

To determine the center of mass of the platform method of hanging was chosen. The method consists in the fact that the examined object is suspended at different points on the fiber. The line, on which the fiber lies, will pass through the center of mass of the object. In our case, we can assume that the robot has symmetry relatively the vertical axis passing through its geometric center. In this case, the center of mass lies on this axis. It remains only to determine the exact position it on the axis. Figures $\mathbf{3 1}$ and $\mathbf{3 2}$ show the results of experiment for determining the center of mass. According to the results it the next value of the desired parameter was determined: $I=0.07 \mathrm{~m}$.

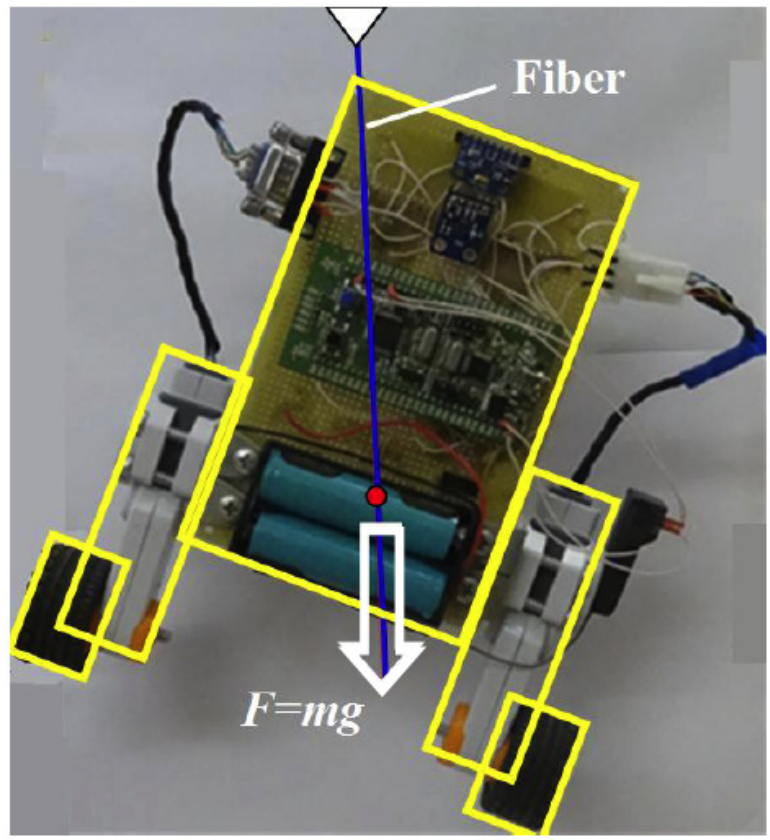

Figure 31: Experiement in determinztion of the distance from the center of mass of the robot to the point of suspension.

To determine the radius of gyration of the platform we use the equation of the period of small oscillations of the physical pendulum:

$T=2 \pi \sqrt{\frac{I}{m g l}}$.

Here $I$ is moment of inertia of the physical pendulum. Taking into account that $I=m \lambda^{2}$, we get:

$\lambda=\frac{T}{2 \pi} \sqrt{g l}$.

The experiment allowed measuring of oscillations of the robot near the lower equilibrium position (the robot was hung upside down). The results are shown in Figure 33. 


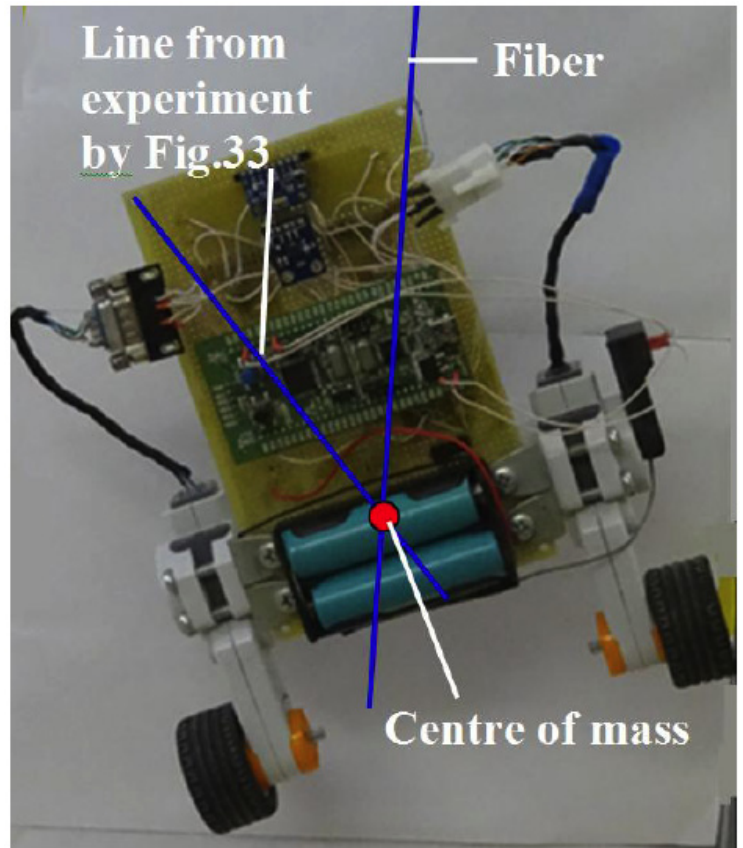

Figure 32: Experiement in determinztion of the distance from the center of mass of the robot to the point of suspension.

By measuring of the period of oscillation, as well as by the previously defined parameter $I$, the following radius of inertia of the robot was determined: $\lambda=0.09$ $m$.

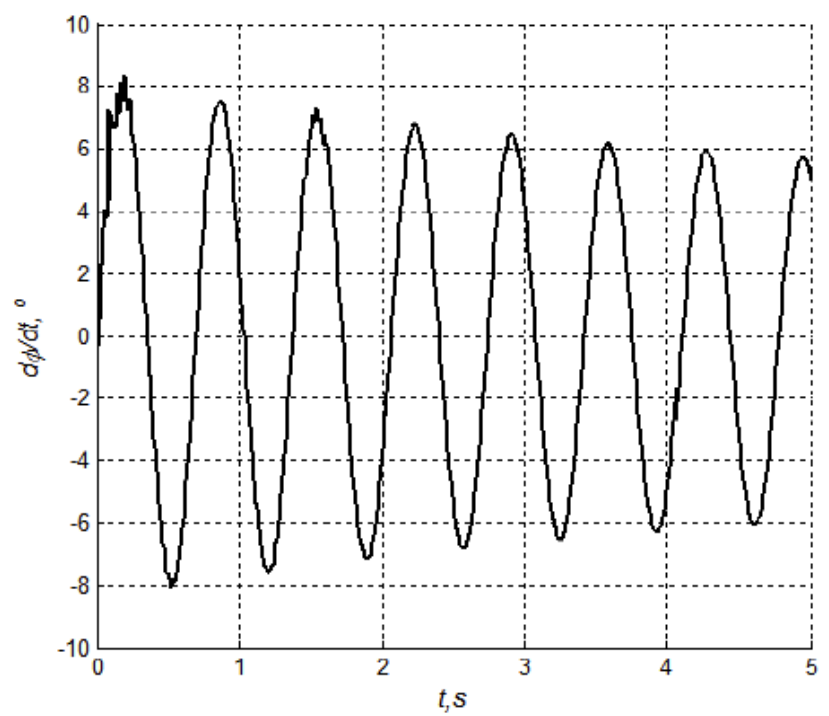

Figure 33: The result of measuring of the moment of inertia of the robot.

To check the correctness of the values of the resulting parameters, as well as the correctness of the previously determined mathematical models of servo motor, following experiment was performed. Fixed voltage was applied to the motor, while the robot was bent at some angle from the vertical. After supplying of voltage to the robot it passed the upper unstable equilibrium position and fell in opposite side. Figure $\mathbf{3 4}$ shows the results of simulations of this experiment and the data obtained in the course of actual experience. Until the initial values of the deflection angle of \pm 10 degrees, the results of the experiment and the simulation are the same with good accuracy. At large angles linearization model begins affecting.

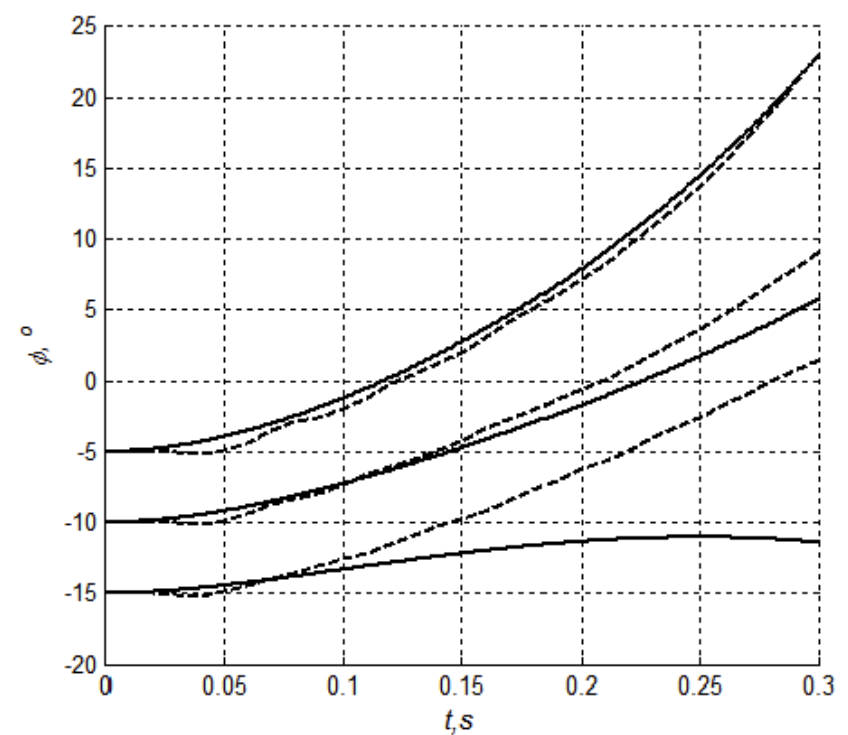

Figure 34: Transient responces in the system when applying a fixed voltage: solid lines are the result of simulation, the broken ones are the result of the experiment.

\section{CALCULATION OF THE CONTROL AND RESULTS}

After getting of all values of the unknown parameters of the model, we can begin calculating the coefficient of the regulator following the procedure described in the first section. First, we must determine harakteric equation of the system with controller:

$$
\begin{aligned}
& A(0)=\operatorname{det}(p I-(A+B K))= \\
& =p^{4}+A_{3}(K) p^{3}+A_{2}(K) p^{2}+A_{1}(K) p+A_{0}(K) \\
& A_{3}(K)=b_{1} k_{1}-b_{2} k_{3}+a_{1}+a_{3}, \\
& A_{2}(K)=b_{1} k_{1}-b_{2} k_{4}-a_{2}, \\
& A_{1}(K)=\left(a_{2} b_{2}-a_{4} b_{1}\right) k_{2}+a_{1} a_{4}-a_{2} a_{3}, \\
& A_{0}(K)=\left(a_{2} b_{2}-a_{4} b_{1}\right) k_{4} .
\end{aligned}
$$

Next, you need to create the desired dynamic equation. For this, we use the results of the experiment described in the previous section. Figure 34 shows that the response time should be at least $0.3 \mathrm{~s}$. Otherwise, the system cannot work. Based on this, we select the following values of the locked system roots:

$\lambda_{1}=\lambda_{2}=\lambda_{3}=\lambda_{4}=-10$. 
These roots values correspond to the following desired equation:

$C(p)=p^{4}+40 p^{3}+600 p^{2}+4000 p+10000$.

Equating the coefficients of terms powers according to equal power of $p$, we obtain the following values for the coefficients:

$k_{1}=1,34 \mathrm{~V} \cdot \mathrm{s} / \mathrm{deg} ; k_{2}=7,6 \mathrm{~V} / \mathrm{deg} ;$

$k_{3}=0,11 \mathrm{~V} \cdot \mathrm{s} / \operatorname{deg} ; k_{4}=0,3 \mathrm{~V} / \mathrm{deg}$.

It should be noted that because of the large number of not accounted non-linearities in the real control object, obtained coefficients may not provide predetermined speed, and in the worst case not even ensure system stability. To verify the regulator, simulation of the processes in a nonlinear system with a calculated controller was accomplished. The simulation results are shown in Figure 35. As can be seen from it, the system is unstable. Therefore, to ensure the efficiency of the device it is necessary to adjust the values of the obtained coefficients.

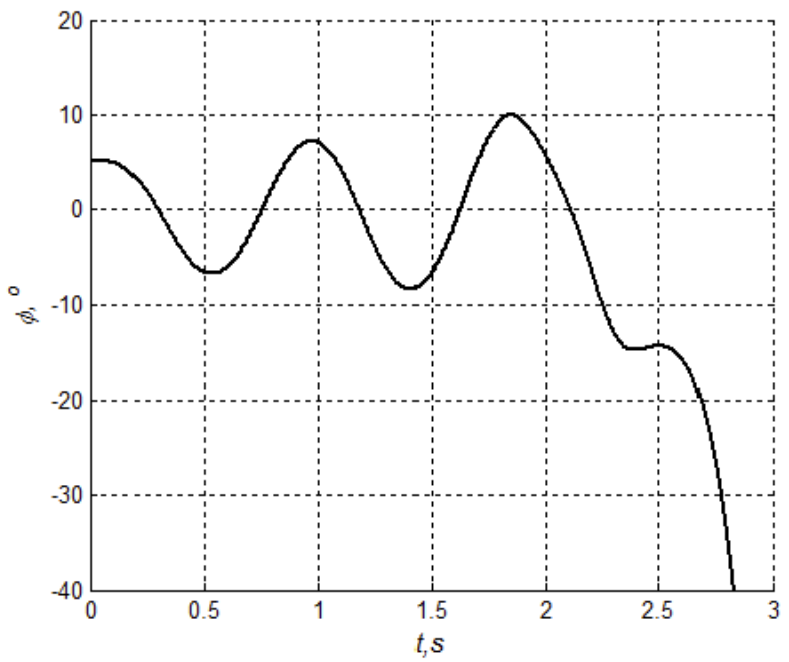

Figure 35: Simulation of the system with the values of the controller coefficients $k_{1}=0.134, k_{2}=0.76, k_{3}=0.011, k_{4}=$ 0.030 .

To adjust the values of coefficients we can use automatic adjustment. Its essence is based on the modeling of processes in the system with different values of the coefficients of the regulator and the estimation of the processes with some quality criterion. In the simplest case, the adjustment of the coefficients is performed in turn one by one. This procedure is similar to the procedure of automatic optimization. However, unlike in optimization, simple tuning does not require optimality of the resulting processes. It is sufficiently to obtain stable processes with acceptable quality. This simplifies the process, and relatively simple algorithm can be used. An example of such an algorithm is shown in Figure 36. The requirements for the dynamic accuracy of the system model are not as stringent as in the case of the optimization.

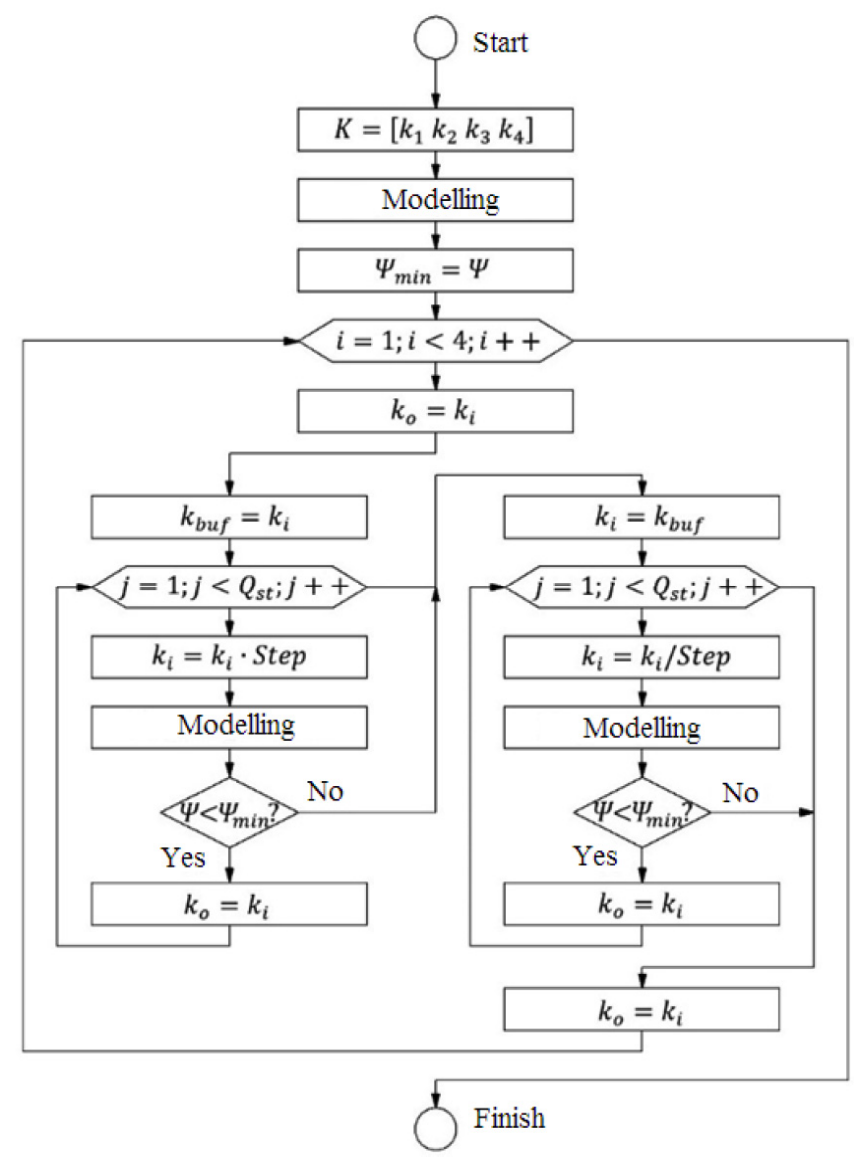

Figure 36: The algorithm of the controller tuning, where $K$ is matrix of coefficients of the controller (regulator), $Q_{s t}$ is the number of steps of the algorithm, Step is step change in the current setting.

To configure the controller the following quality criteria has been selected:

$\psi(T)=\int_{0}^{T} t|e(t)| d t$

Here, $e(t)$ is error of control. The factor $t$ introduces weight of the errors over time, increasing its contribution to the cost function in the end of the process. The result of the procedure yielded the following values for the coefficients:

$k_{1}=0,086 \mathrm{~V} \cdot \mathrm{s} / \mathrm{deg} ; k_{2}=3,96 \mathrm{~V} / \mathrm{deg}$;

$k_{3}=0,023 \mathrm{~V} \cdot \mathrm{s} / \mathrm{deg} ; k_{4}=0,055 \mathrm{~V} / \mathrm{deg}$.

Figure 37 shows simulated transient processesof the system compared with ones in the real device. The system is stabilized in the both cases. Tests have shown that with given values of the coefficients of the 
controller, robot is able to work off the initial perturbations about angle of inclination within $5-7^{\circ}$.

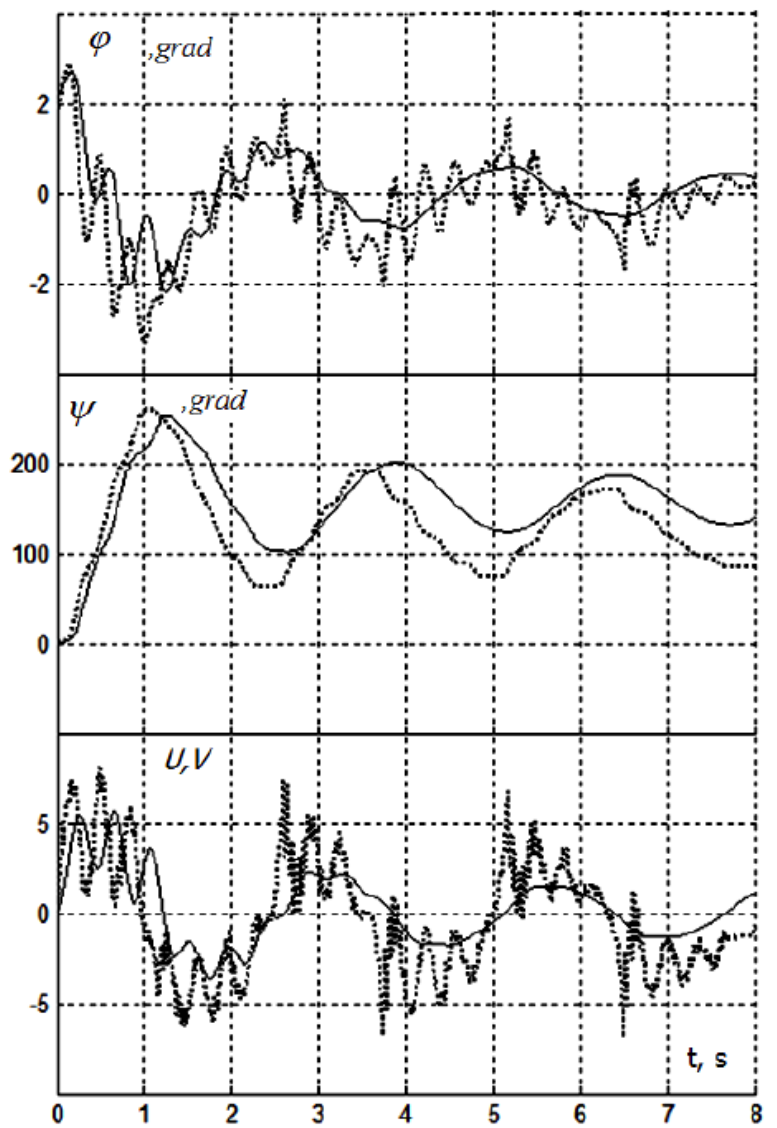

Figure 37: The result of the simulation of the system and the result of the real system working with the values of the coefficients of the regulator $k_{1}=0.086 ; k_{2}=3.96 ; k_{3}=0.023$; $k_{4}=0.055$; solid line is modeling, dotted line is real system.

According to the results of the work it can be concluded that the modal method does not always produce acceptable results for the object "inverted pendulum", to this the balancing robot. This is due to the fact that the calculations used a linearized model. Real object has a number of non-linearities which degrade the system quality. However, used principle of feedback and regulator structure a good even for the non-linear object. For the final tuning it is better to use the automatic tuning procedure on the basis of the full non-linear model of the system.

The site of the Department of Automation of the Novosibirsk State Technical University [5] presents the video about the working of the balancing robot, described in this article. It is enough to click on the center of the image that appears on the site below. This image is shown in Figure $\mathbf{3 8}$.

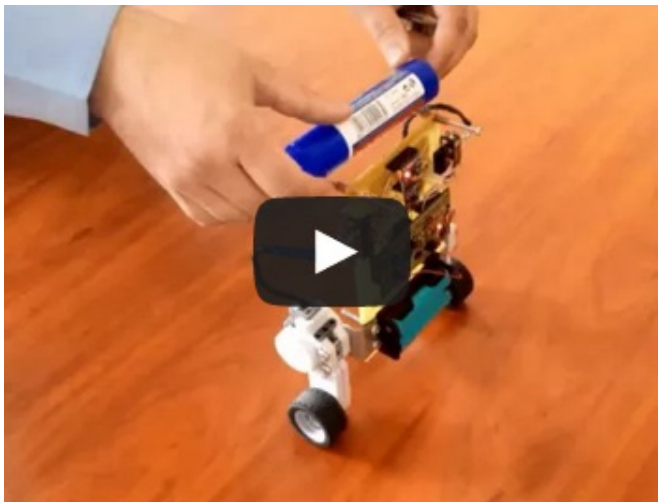

Figure 38: The window on the website [5], to which you can press to view a video about its working.

The work was supported by the Russian Ministry of the state, Project № 2014/138, the theme of the "New structures, models and algorithms for the control of breakthrough technology systems based on high-tech results of intellectual activity".

\section{REFERENCES}

[1] MPU-6000 and MPU-6050 Register Maps and Descriptions [Electronic resource]. - Acess data: http://www. invensense.com/products/motion-tracking/6-axis/mpu$6050 /$

[2] Zadacha sinteza $v$ teorii regulirovanija: ucheb. posobie / A.S. Vostrikov. - Novosibirsk: Izd-vo NGTU, 2011. - 104 s.

[3] Chto merjaet akselerometr [Electronic resource]. - Acess data: http://dpla.ru/acclmmer.img/acclmmer.htm

[4] MJeMS-giroskopy - edinstvo vybora [Electronic resource]. Acess data: http://www.electronics.ru/journal/article/512

[5] Web site of the Department of Automation in Novosibirsk State Technical University, page "Sceince work". URL: http://ait.cs.nstu.ru/content/nauchnaja-dejatelnost

(c) 2015 Fedorov et al.; Licensee Lifescience Global.

This is an open access article licensed under the terms of the Creative Commons Attribution Non-Commercial License (http://creativecommons.org/licenses/by-nc/3.0/) which permits unrestricted, non-commercial use, distribution and reproduction in any medium, provided the work is properly cited. 